\title{
An Improved Picard Iteration Scheme for Simulating Unsaturated Flow in Layered Porous Media
}

\author{
S.R. Zhu
}

Chengdu University of Technology

L.Z.Wu (D13880936151@163.com)

Chengdu University of Technology

S.H. Li

Chengdu University of Technology

\section{Research Article}

Keywords: Unsaturated flow, Picard iteration, Layered porous media, Control volume method, Multistep preconditioner, Non-uniform multigrid

Posted Date: May 25th, 2021

DOl: https://doi.org/10.21203/rs.3.rs-508570/v1

License: @ (i) This work is licensed under a Creative Commons Attribution 4.0 International License. Read Full License

Version of Record: A version of this preprint was published at Arabian Journal of Geosciences on November 18th, 2021. See the published version at https://doi.org/10.1007/s12517-021-08497-y. 
An improved Picard iteration scheme for simulating unsaturated

\title{
flow in layered porous media
}

\author{
S. R. Zhu ${ }^{\text {a }}$ L. Z. Wu ${ }^{\text {a* }}$ S. H. Li ${ }^{\text {a }}$
}

a) State Key Laboratory of Geohazard Prevention and Geoenvironment Protection, Chengdu University of Technology, 610059, Chengdu, Sichuan, P. R. China

*corresponding author: Email: wulizhou07@.cdut.edu.cn

Submitted on May 8, 2021 


\begin{abstract}
Picard iteration method is commonly used to obtain numerical solution of unsaturated flow in porous media. However, because the system of linear equations derived from Richards equation is seriously ill-conditioned, Picard iteration has slow convergence rate and low computational efficiency, particularly in layered porous media. In this study, control volume method based on non-uniform nodes is used to discrete Richards equation. To improve the convergence rate of Picard iteration, we combine the non-uniform multigrid correction method with the multistep preprocessing technology. Thus, an improved Picard iteration scheme with multistep preconditioner based on non-uniform multigrid correction method (NMG-MPPI $(m))$ is proposed to model 1D unsaturated flow in layered porous media. Three test cases were used to verify the proposed schemes. The result shows that the condition number of the coefficient matrix has been greatly reduced using the multistep preconditioner. Numerical results indicate that NMG-MPPI $(m)$ can solve Richards equation at a faster convergence rate, with higher calculation accuracy and good robustness. Compared with conventional Picard iteration, NMG-MPPI $(m)$ shows a very high speed-up ratio. As a result, the improved Picard iteration scheme has good application for simulating unsaturated flow in layered porous media.
\end{abstract}

Keywords: Unsaturated flow; Picard iteration; Layered porous media; Control volume method; Multistep preconditioner; Non-uniform multigrid; 


\section{Introduction}

Water is one of the most important factors affecting the slope stability (Jiang et al. 2016; Wu et al. 2017; Mishra et al. 2020). Water infiltration changes unsaturated zones, and decreases shear strength, thus plays a key role in slope stability (Zhou et al. 2018; Sihag et al. 2019; Cross et al. 2020). Richards' equation (RE) (Richards 1931) is often used to describe unsaturated seepage processes (Hashemi Jokar et al. 2019; Wu et al. 2020a). Due to the nonlinear nature of RE, the analytical solution is difficult to obtain, and it is often obtained based on an exponential form (Srivastava and Yeh 1991; Tracy 2006). Thus, different numerical methods were employed to solve RE (Wu et al. 2016; Zeng et al. 2018), including finite volume method (Patankar 1980), finite difference method (Wang and Anderson 1982) and finite element method (Šimůnek et al. 2009). Wang and Anderson (1982) introduced the practical application of finite difference and finite element to the numerical simulation of groundwater seepage and pollution propagation. In Software HYDRUS-1D, the standard Galerkin-type linear finite element schemes were applied to numerically solve RE (Šimůnek et al. 2009). ChávezNegrete et al. (2018) proposed a generalized finite difference method for solving RE using non-rectangular structural grid. Hosseini et al. (2020) proposed an extended finite element method to model density-driven solute transport in fractured porous media. Besides, control volume method was used to simulate heat transfer and fluid flow (Patankar 1980). Svyatskiy and Lipnikov (2017) proposed a second-order accurate finite volume scheme to investigate RE, which was solved using high-order upwind algorithms for the relative permeability. Of course, other advanced numerical methods 
exhibit attractive accuracy, computational efficiency, or ease of implementation under certain conditions in solving variable saturated flow problems. These include mixed hybrid finite elements method/finite volumes (Abderahim and Mohammed 2011), adaptive domain decomposition (Kuraz et al. 2014), finite analytic method (Zhang et al. 2016), and Chebyshev spectral method (Wu et al. 2020b).

Generally, a system of linearized algebraic equations can be obtained by using above numerical methods to discretize RE. Nevertheless, it needs to be re-evaluated for each iteration (Šimůnek et al. 2009). For this reason, Newton-based nonlinear iterative schemes are usually used, including lower precision approximations such as the Picard iteration (PI) (Lott et al. 2012). Since hydraulic conductivity and water content are nonlinear functions of the pressure head, the numerical results of RE using PI show a low reliability. Therefore, Celia et al. (1990) proposed a modified Picard iteration to ensure the mass conservation in the temporal discretization. This modified Picard iteration has been applied to HYDRUS-1D (Šimůnek et al. 2009). Recently, Zha et al. (2017) made further improvements based on the algorithms proposed by Celia et al. (1990) to overcome the numerical non-convergence for simulating infiltration into extremely dry soil. However, these improvements are limited to the overall mass balance of the RE, and do not improve the convergence rate of the solutions.

As PI has poor convergence and is time-consuming, increasing attention has focused on improving its convergence rate (Lott et al. 2012; List and Radu, 2016; Illiano et al. 2020). Among them, the use of preprocessing techniques in the iterative process can improve the convergence rate. Usually, the system of linear equations at each iteration 
is preprocessed to improve the ill-condition of the equations (Benzi 2002). For example, a class of parallel multistep successive preconditioning strategies were proposed to improve the computational efficiency and stability of the solution process for systems of linear equations (Wang and Zhang 2003). the multi-grid correction method was proposed to quickly eliminate iteration errors, thereby achieving a faster convergence rate (Briggs et al. 2000). The Chebyshev semi-iterative method with polynomial preconditioner was proposed to effectively improve the convergence rate in solving the linearized Richards equation (Zhu et al. 2020). Thus, to enhance the computational efficiency of PI, an improved Picard iteration with multistep preconditioner based on non-uniform multigrid correction method (NMG-MPPI $(m)$ ) was proposed to simulate unsaturated flow in layered unsaturated soils.

The purpose of this paper is to develop a new iteration method to effectively solve the problems of unsaturated flow in porous media. First, NMG-MPPI $(m)$ is applied to investigate transient flow in porous media. The proposed method was verified by three examples of unsaturated flow. The convergence rate, calculation efficiency, calculation accuracy, and robustness of the proposed schemes are compared and discussed against the numerical results of conventional iteration methods.

\section{Numerical Procedures}

\subsection{Governing Equation and Discretization}

Richards' equation is often used to describe transient flow in porous media (Wu et al. 2020b), which is expressed as in a 1D form: 


$$
\frac{\partial}{\partial z}\left[K_{z}(h)\left(\frac{\partial h}{\partial z}+1\right)\right]=\frac{\partial \theta}{\partial t}
$$

where $z$ is the vertical coordinate, as represented in Fig. $1 ; h$ represents the pressure head; $\theta$ and $K_{z}(h)$ denote moisture content and hydraulic conductivity along the $z$-axis, respectively, which are both functions of the pressure head $(h)$;

Control volume method is used to discretize Eq. (1). For two-layer soils, the nonuniform grid is adopted in Fig. 1, and its coordinate can be expressed as:

$$
\begin{aligned}
& z_{1 i}=\cos \left(i \pi / N_{1}\right) \times \frac{L 1}{2}+\frac{L 1}{2}, \quad i=N_{1}, N_{1}-1, \mathrm{~K} 0 \\
& z_{2 i}=\cos \left(i \pi / N_{2}\right) \times \frac{L 2}{2}+\frac{L 2}{2}+L 1, \quad i=N_{2}-1, N_{2}-2, \mathrm{~K} 0
\end{aligned}
$$

where $L_{1}$ and $L_{2}$ are the height of soil layers 1 and 2, respectively; $i$ denotes non-uniform grid nodes; and $N_{1}$ and $N_{2}$ represent the number of nodes in layers 1 and 2, respectively. To ensure the mass balance in the temporal discretization, a modified Picard iteration algorithm for $\partial \theta / \partial t$ is proposed as follows (Celia et al. 1990):

$$
\frac{\partial \theta}{\partial t}=C^{j+1, k} \frac{h^{j+1, k+1}-h^{j+1, k}}{\Delta t}+\frac{\theta^{j+1, k}-\theta^{j}}{\Delta t}
$$

where $C$ is the specific moisture capacity, defined as $C(h)=\partial \theta / \partial h ; \Delta t$ denotes the time step; and the superscripts $j$ and $k$ represent the time level and iteration level, respectively. Eq. (3) has been widely used in commercial software including HYDRUS (Šimůnek et al. 2009; Zha et al. 2017). Since the control volume of node $i$ at the interface contains two types of soils (Fig. 1), the moisture content at the interface needs to be defined as (Zha et al. 2013):

$$
\theta_{i}=\frac{0.5 \Delta z_{i-1} \theta\left(h_{i}, \text { parameter1 }\right)+0.5 \Delta z_{i} \theta\left(h_{i}, \text { parameter } 2\right)}{0.5\left(\Delta z_{i-1}+\Delta z_{i}\right)}
$$


where $\Delta z_{i-1}$ and $\Delta z_{i}$ represent the lengths between adjacent nodes (Fig. 1); and $\theta$ $\left(h_{i}\right.$, parameter 1$)$ and $\theta\left(h_{i}\right.$, parameter 2$)$ denote the moisture content obtained using the parameters of layers 1 and 2, respectively.

The discretized equation is now derived by integrating Eq. (1) over the control volume in Fig. 1 and over the time interval from $t$ to $t+\Delta t$ (Patankar 1980). Thus,

$$
\int_{i_{W}}^{i_{E}} \int_{t}^{t+\Delta t} \frac{\partial \theta}{\partial t} d t d z=\int_{t}^{t+\Delta t} \int_{i_{W}}^{i_{E}} \frac{\partial}{\partial z}\left[K_{z}(h)\left(\frac{\partial h}{\partial z}+1\right)\right] d z d t
$$

Substituting Eq. (3) into Eq. (5), we can obtain

$$
\begin{aligned}
& C_{i}^{j+1, k} \frac{h_{i}^{j+1, k+1}-h_{i}^{j+1, k}}{\Delta t}(\Delta z)_{i}+\frac{\theta_{i}^{j+1, k}-\theta_{i}^{j}}{\Delta t}(\Delta z)_{i}=\frac{K_{i_{E}}^{j+1, k}\left(h_{i+1}^{j+1, k+1}-h_{i}^{j+1, k+1}\right)}{(\delta z)_{E}} \\
& -\frac{K_{i_{W}}^{j+1, k}\left(h_{i}^{j+1, k+1}-h_{i-1}^{j+1, k+1}\right)}{(\delta z)_{W}}+K_{i_{E}}^{j+1, k}-K_{i_{W}}^{j+1, k}
\end{aligned},
$$

where

$$
\begin{gathered}
K_{i_{E}}=\frac{2 K_{i} K_{i+1}}{K_{i}+K_{i+1}} \\
K_{i_{W}}=\frac{2 K_{i} K_{i-1}}{K_{i}+K_{i-1}}
\end{gathered}
$$

If $i$ is at the interface, $K_{i_{E}}$ is obtained using the parameters of layer 2; while $K_{i_{W}}$ is obtained using the parameters of layer 1 .

For a steady-state infiltration, Eq. (6) can be simplified as:

$$
\frac{K_{i_{E}}^{k}\left(h_{i+1}^{k+1}-h_{i}^{k+1}\right)}{(\delta z)_{E}}-\frac{K_{i_{W}}^{k}\left(h_{i}^{k+1}-h_{i-1}^{k+1}\right)}{(\delta z)_{W}}+K_{i_{E}}^{k}-K_{i_{W}}^{k}=0 .
$$

\subsection{Picard iteration scheme}

According to Eqs. (6) and (8), the matrix format of Picard iteration based on nonuniform grid (N-PI) can be written as follows: 


$$
\begin{aligned}
\mathbf{A}\left(\mathbf{h}^{j+1, k}\right) \mathbf{h}^{j+1, k+1} & =\mathbf{f}\left(\mathbf{h}^{j+1, k}, \mathbf{h}^{j}\right) . \\
\mathbf{A}\left(\mathbf{h}^{k}\right) \mathbf{h}^{k+1} & =\mathbf{f}\left(\mathbf{h}^{k}\right) .
\end{aligned}
$$

where $\mathbf{A}$ is a symmetrical tridiagonal matrix; and $\mathbf{h}$ and $\mathbf{f}$ are both a vector.

A system of linear algebraic equations is first derived at each iteration, and then a basic linear iterative method, such as Gaussian elimination, conjugate gradient method or Gauss-Seidel method, is used to solve the system of linear equations (Šimůnek et al. 2009). After solving Eqs. (9) - (10) for the first time, the coefficients in Eqs. (9) - (10) are re-evaluated using the first solution, and the new linear equations are solved again. The iteration procedure terminates when the iteration error is satisfied as follows:

$$
\frac{\left\|\mathbf{h}^{j, k+1}-\mathbf{h}^{j, k}\right\|_{\infty}}{\left\|\mathbf{h}^{j, k}\right\|_{\infty}}<\varepsilon
$$

where $\varepsilon$ is the tolerance, and set to $10^{-10}$ in this study.

\subsection{Mathematical models of SWCC}

There are many models to describe the functions $\theta$ and $K(h)$ (Liu et al. 2015; Zhang et al. 2016). Among them, an exponential function is a classical model (Gardner 1958), which is expressed as:

$$
\begin{gathered}
\theta(h)=\theta_{r}+\left(\theta_{s}-\theta_{r}\right) e^{\alpha h}, \\
K(h)=K_{s} e^{\alpha h},
\end{gathered}
$$

where $K_{s}$ is the saturated hydraulic conductivity; $\theta_{s}$ and $\theta_{r}$ represent the saturated and residual moisture contents, respectively; and $\alpha$ is the fitting parameter.

In addition, another model can be often written as (van Genuchten 1980),

$$
\theta(h)=\theta_{r}+\left(\theta_{s}-\theta_{r}\right)\left[1+(\beta|h|)^{n}\right]^{-m},
$$




$$
K(h)=K_{s} S_{e}^{\frac{1}{2}}\left[1-\left(1-S_{e}^{\frac{1}{m}}\right)^{m}\right]^{2}
$$

where $S_{e}$ represents the effective saturation, defined as $S_{e}=1 /\left[1+(\beta|h|)^{n}\right]^{m} ; \beta$ and $n$ are both the fitting parameters; and $m=1-1 / n$;

\section{Improved Picard iteration scheme}

In general, PI has only a first-order convergence rate, and its calculation efficiency is low. To improve the convergence rate of PI, a multistep preconditioner strategy is introduced. Firstly, the symmetric Gauss-Seidel (SGS) preconditioner $\mathbf{M}_{S G S}$ is given by:

$$
\mathbf{M}_{S G S}=(\mathbf{D}-\mathbf{L}) \mathbf{D}^{-1}(\mathbf{D}-\mathbf{U})
$$

where $\mathbf{D}$ represents diagonal matrix of $\mathbf{A} ; \mathbf{L}$ and $\mathbf{U}$ represent the lower triangular matrix and the upper triangular matrix of $\mathbf{A}$, respectively.

Furthermore, the multistep preconditioner strategy considering the symmetric GaussSeidel preconditioner can be described as follows:

Algorithm MP(m). multistep preconditioner

Given the number of steps $m>0$, the coefficient matrix $\mathbf{A}$, and the filter parameter $\varepsilon$

Let $\mathbf{A}_{1}=\mathbf{A}$

For $(i=1 ; i \leq m ; i++)$

Compute a SGS preconditioner $\mathbf{M}_{S G S}^{i}$ using $\mathbf{A}_{i}$

Compute $\quad \mathbf{A}_{i+1}=\mathbf{M}_{S G S}^{i} \mathbf{A}_{i}$

End 
Drop small entries of $\mathbf{M}_{S G S}^{m}$ with respect to $\tau$

$\prod_{i=1}^{m} \mathbf{M}_{S G S}^{i}$ is the preconditioner for Eqs. (9) - (10)

In this study, $\tau$ is uniformly set to $10^{-4}$. Thus, the Picard iteration with multistep preconditioner based on non-uniform grid $(\mathrm{N}-\mathrm{MPPI}(m))$ can be expressed as:

$$
\prod_{i=1}^{m} \mathbf{M}_{S G S}^{i}\left(\mathbf{h}^{j+1, k}\right) \mathbf{A}\left(\mathbf{h}^{j+1, k}\right) \mathbf{h}^{j+1, k+1}=\prod_{i=1}^{m} \mathbf{M}_{S G S}^{i}\left(\mathbf{h}^{j+1, k}\right) \mathbf{f}\left(\mathbf{h}^{j+1, k}, \mathbf{h}^{j}\right)
$$

It can be easily seen here that Eq. (17) degenerates into Eq. (9) when $m=0$. That is, no preprocessing is performed. After multi-step preprocessing, the condition number of the coefficient matrix in Eqs. (9) - (10) can be greatly reduced, and then the multigrid method is used to further improve calculation efficiency and accuracy.

A non-uniform multigrid correction $\mathrm{V}$-Cycle scheme (NMG) is proposed to improve the calculation efficiency of N-MPPI $(m)$ (Fig. 2). More simply, the non-uniform twogrid correction scheme is used to illustrate NMG. Assuming that the solution vectors of the fine and coarse grids are $\mathbf{h}^{f}$ and $\mathbf{h}^{c}$, respectively. The following two-grid correction scheme is used:

(i) Relax $\mu$ times on Eq. (17) for the non-uniform fine grid with initial vector $\mathbf{h}^{f 0}$.

(ii) Compute the non-uniform fine-grid residual $\mathbf{r}^{f}=\mathbf{f}\left(\mathbf{h}^{f \mu}\right)-\mathbf{A}\left(\mathbf{h}^{f \mu}\right) \mathbf{h}^{f \mu}$ and restrict it to the non-uniform coarse grid by $\mathbf{r}^{c}=\mathbf{R} \mathbf{r}^{f}$, and $\mathbf{h}^{c}=\mathbf{R} \mathbf{h}^{f \mu}$, where $\mathbf{R}$ is the restriction operator from fine grid to coarse grid.

(iii) Solve the following equation to obtain vector $\mathbf{h}^{c 1}$ for the non-uniform coarse grid:

$$
\mathbf{A}\left(\mathbf{h}^{c}\right) \mathbf{h}^{c 1}=\mathbf{A}\left(\mathbf{h}^{c}\right) \mathbf{h}^{c}+\mathbf{r}^{c}
$$

(iv) Interpolate the coarse-grid error $\left(\mathbf{h}^{c 1}-\mathbf{h}^{c}\right)$ to the fine grid and correct the fine- 
grid approximation solution by:

$$
\mathbf{h}^{f \mu} \leftarrow \mathbf{h}^{f \mu}+\mathbf{I}\left(\mathbf{h}^{c 1}-\mathbf{h}^{c}\right)
$$

where $\mathbf{I}$ is the interpolation operator from coarse to fine grids.

(v) Relax $\mu$ times on Eq. (17) for the non-uniform fine grid with corrected vector $\mathbf{h}^{f \mu}$, where $\mu$ usually takes a small natural number.

If $\mathbf{h}^{f \mu}$ converges according to Eq. (11), then stop; otherwise, go to step (i). Steps (i) and (v) can eliminate the high-frequency components of the iteration error, while steps (ii)- (iv) can eliminate the remaining low-frequency smooth components. Through multiple cycles of the above steps, it is expected to eliminate the iteration error as soon as possible.

As can be seen from the two-grid correction scheme, NMG requires correspondence between the non-uniform grids of each layer (Fig. 2), which needs to further define the interpolation (I) and restriction $(\mathbf{R})$ operators. In Fig. 2, the restriction operator for nodes $i$ can be expressed as:

$$
u_{i}=\frac{(\Delta z)_{1}}{(\Delta z)_{i}} u_{2 i-1}+\frac{(\Delta z)_{2}}{(\Delta z)_{i}} u_{2 i}+\frac{(\Delta z)_{3}}{(\Delta z)_{i}} u_{2 i+1}
$$

The interpolation operator can be written as:

$$
\begin{gathered}
u_{2 i}=u_{i} \\
u_{2 i+1}=\frac{(\Delta z)_{2}}{(\Delta z)_{1}+(\Delta z)_{2}} u_{i}+\frac{(\Delta z)_{1}}{(\Delta z)_{1}+(\Delta z)_{2}} u_{i+1} \\
u_{2 i+2}=u_{i+1}
\end{gathered}
$$

Furthermore, the two-grid correction scheme is used recursively to obtain a more practical multigrid correction scheme. A brief flowchart of Picard iteration with the 
multistep preconditioner based on the non-uniform multigrid correction scheme (NMG$\operatorname{MPPI}(m))$ is shown in Fig. 3. To verify the computational efficiency of the proposed schemes, the speed-up ratio is defined as:

$$
S_{N-P I / N M G-M P P I(m)}=\frac{T_{N-P I}}{T_{N M G-M P P I(m)}},
$$

where $T_{N-P I}$ is the running time for N-PI; and $T_{N M G-M P P I(m)}$ is the running time for NMG-MPPI $(m)$. Based on the proposed schemes, all numerical simulations were run on MATLAB (version: R2014a) on an Intel i7-4720 CPU.

\section{Numerical Tests}

\subsection{Test 1}

This test simulates the $1 \mathrm{D}$ transient flow in homogeneous porous media to verify the effectiveness of the proposed schemes. The mathematical model is a shown in Fig. 1. A uniform soil layer without interfaces is assumed. The thickness $(L)$ of the soil layer is $10 \mathrm{~m}$. Gardner model is used to represent the soil-water characteristic curve of unsaturated soils, and the parameters are $\alpha=3.2 \times 10^{-4} / \mathrm{m}, \theta_{s}=0.35, \theta_{r}=0.14$, and $K_{s}=1 \times 10^{-4} \mathrm{~m} / \mathrm{s}$. The governing equation is described using Eq. (1). The initial condition is assumed to be $h(z, t=0)=h_{d}$. The boundary conditions of this model are set as:

$$
\begin{gathered}
h(z=0)=h_{d}, \\
h(z=10 m)=0 .
\end{gathered}
$$

where $h_{d}$ represents the pressure head of dry soil, defined as $h_{d}=-10^{3} \mathrm{~m}$. An analytical solution to the transient flow in unsaturated soils was given by (Tracy 2006), 


$$
h_{\text {ana }}(z, t)=\frac{1}{\alpha} \log \left(h_{t}^{*}(z, t)+h_{s}^{*}(z)+e^{\alpha h_{d}}\right)
$$

where

$$
\begin{gathered}
h_{s}^{*}(z)=\left(1-e^{\alpha h_{d}}\right) \times\left(1-e^{-\alpha z}\right) /\left(1-e^{-\alpha L}\right), \\
h_{t}^{*}(z, t)=\frac{2\left(1-e^{\alpha h_{d}}\right)}{L c} e^{\alpha(L-z) / 2} \sum_{m=1}^{\infty}(-1)^{m}\left(\frac{\lambda_{m}}{\mu_{m}}\right) \sin \left(\lambda_{m} z\right) e^{-\mu_{m} t},
\end{gathered}
$$

with $c=\alpha\left(\theta_{s}-\theta_{r}\right) / K_{s}, \lambda_{m}=k \pi / L$, and $\mu_{m}=\left(\alpha^{2} / 4+\lambda_{m}^{2}\right) / c$.

Furthermore, the numerical solution is compared with the analytical solution $\left(\mathbf{h}_{\text {ana }}\right)$, and the relative error is defined as $\left|\left(\mathbf{h}_{\text {ana }}-\mathbf{h}\right) / \mathbf{h}_{\text {ana }}\right| \times 100 \%$. The total simulation time is 5 hours. The convergence rates of different iterative methods are shown in Fig. 4. The parameter $\mu$ is 3 , the time step is $0.1 \mathrm{~h}$, and the number of nodes is 129 . It can be seen that the convergence rate of N-PI is the slowest. Both N-MPPI $(m)$ and NMG-MPPI $(m)$ have a significant improvement in the convergence rates as $m$ increases. Among them, NMG-MPPI $(m)$ has the fastest convergence rates. The condition number of the coefficient matrix can be greatly reduced (Fig. 5(a)) as $m$ increases. However, the calculation accuracy of NMG-MPPI $(m)$ is affected by $m$ (Fig. 5(b)).

Table 1 summarizes the average iterations for each time step, relative error, and running time at different time steps and number of nodes using different schemes. The time steps are taken to be $0.1 \mathrm{~h}, 0.05 \mathrm{~h}$, and $0.025 \mathrm{~h}$, respectively. And the number of nodes are set to 97,129 , and 161 . It can be easily seen that average iterations and running time of NMG-MPPI $(m)$ with $m=2$ is the least under same conditions. And the maximum relative errors of NMG-MPPI(2) are smaller than N-PI and N-MPPI(1). Besides, relative errors and average iterations decrease as decreasing time step for the 
constant number of nodes; and the running time is the opposite. For the same time step, the average iterations and running time increase with increasing number of nodes; and the maximum relative errors decrease with the increase of the number of nodes. Meanwhile, the numerical results obtained by NMG-MPPI(2) are very consistent with the analytical solutions as shown in Fig. 6. The results also show that the speed-up ratios of N-MPPI(1) relative to N-PI is greater than 1.7 (Fig. 7(a)). And the minimum speedup ratio of NMG-MPPI(2) relative to N-PI is greater than 2.8 (Fig. 7(b)). This test indicates that the proposed NMG-MPPI $(m)$ effectively simulates transient flow in unsaturated soils. The results show that NMG-MPPI $(m)$ with $m=2$ is the best in terms of computational efficiency and accuracy.

\subsection{Test 2}

\subsubsection{Two - layer soils}

For two-layer soils, the ill-condition of the coefficient matrix is extremely serious due to the different physical parameters of the soil layers (Liu et al. 2015). This test simulates the 1D steady-state flow in two-layer unsaturated soils to further verify the effectiveness of the proposed NMG-MPPI $(m)$. The mathematical model is shown in Fig. 1, where $L_{1}=L_{2}=5 \mathrm{~m}$. Its parameters are set to $\alpha=8 \times 10^{-3} / \mathrm{m}$ (Liu et al. 2015), and $\mu=15$. Let the number of nodes $N_{1}=N_{2}=48$. In Table 2 , the hydraulic conductivity of soil layer 1 is $10^{-1} \mathrm{~m} / \mathrm{s}$, and the hydraulic conductivity of soil layer 2 ranges from $10^{-2}$ to $10^{-9} \mathrm{~m} / \mathrm{s}$. This dramatic change of hydraulic conductivity may result in the illconditioned linear equations. Moreover, the governing equation is Eq. (8). The boundary conditions of this model are consistent with those in Test 1; and the initial 
conditions is $h(z)=0$.

Fig. 8 shows the condition number of the coefficient matrix with and without using the MP algorithm for Cases 1 to 8 . It can be found that the condition number of the matrix using $\mathrm{MP}(2)$ has been greatly reduced. The numerical results also show that NMG-MPPI(2) can converge to obtain a numerical solution in different Cases (Fig. 9(a) - (c)). Fig. 10(a) represents the iterations of different methods. It can be found that the proposed NMG-MPPI(2) has the fastest convergence rate compared with N-PI and NMPPI(1). The improved NMG-MPPI(2) shows a more significant acceleration effect for Cases 1 to 8 than N-PI and N-MPPI(1) (Fig. 10(b)).

\subsubsection{Three - layer soils}

Similar to the two-layer soils, the linear equations for the three-layer soils are also ill-conditioned. This test can further verify the effectiveness of the proposed schemes. The mathematical model is shown in Fig. 11, where $L_{1}=L_{3}=4 \mathrm{~m}$, and $L_{2}=2 \mathrm{~m}$. In this test, $N_{1}=N_{3}=64$, and $N_{2}=32$. The setting of the hydraulic conductivity of the threelayer soils is shown in Table 3. The boundary conditions can be expressed as:

$$
\begin{gathered}
h(z=0)=0, \\
h(z=10 m)=-1000 m .
\end{gathered}
$$

Other conditions remain unchanged with those of the two-layer soils. Fig. 12 shows the condition number of the coefficient matrix with and without using the MP algorithm for Cases 9 to 13 . When the MP algorithm is used, the condition number is greatly reduced. Particularly using MP(2), the condition number is reduced to the order of $10^{3}$ for Cases 9 to 13 . The numerical results show that NMG-MPPI(2) can rapidly obtain a 
numerical solution in Fig. 13(a)-(b). Compared with N-PI and N-MPPI(1), NMGMPPI(2) has few iterations (Fig. 14(a)) and fast acceleration (Fig. 14(b)). This test indicates that the proposed schemes can well improve the ill-condition of system of linear equations in layered soils and obtain numerical solutions effectively.

\section{Application Examples}

In this example, we investigate 1D transient flow into the two-layer soil slopes. And van Genuchten model is considered here. In Fig. 15, the thickness of soil layer $1\left(L_{1}\right)$ and soil layer $2\left(L_{2}\right)$ are $3 \mathrm{~m}$ and $2 \mathrm{~m}$, respectively; and the slope angle $(\varphi)$ is 33 degrees. Besides, Eq. (1) needs to be modified to suit water infiltration of slopes (Iverson 2000; Wu et al. 2020b):

$$
\frac{\partial}{\partial z}\left[K_{z}(h)\left(\frac{\partial h}{\partial z}+\cos \varphi\right)\right]=\frac{\partial \theta}{\partial t} .
$$

where $\varphi$ denoting the slope angle. If the thickness of the landslide is much smaller than the slope length, the infinite slope theory can be commonly used in the stability analysis of shallow landslides. The safety factor $\left(F_{S}\right)$ can be expressed as (Iverson 2000):

$$
F_{S}=\frac{c^{\prime}+\left(L \gamma_{t} \cos ^{2}(\varphi)-h \gamma_{w} \cos ^{2}(\varphi)\right) \tan \phi^{\prime}}{L \gamma_{t} \cos (\varphi) \sin (\varphi)},
$$

where $c^{\prime}$ represents the effective cohesion; $\phi^{\prime}$ represents the effective friction angle; $\gamma_{t}$ and $\gamma_{w}$ denote the unit weights of soil and water, respectively, and $L$ denotes the total thickness of the two-layer soil slopes.

The numerical solutions $(h(z, t))$ obtained using the proposed schemes is substituted into Eq. (31) for the slope stability analysis. The parameters for two soil textures are 
shown in Table 4 (Zha et al. 2013). The bottom boundary condition is groundwater table, defined as $h(z=0, t)=0$. The top boundary condition is given by ( Wu et al. 2016):

$$
\begin{aligned}
& {\left[K \frac{\partial h}{\partial z}+K\right]_{z=L 1+L 2}=q_{t}, \quad t<t_{I},} \\
& h_{z=L 1+L 2}=h_{0}, \quad t \geq t_{I},
\end{aligned}
$$

where $t_{I}$ represents ponding time; $q_{t}$ denotes the rainfall flux at the soil surface when the rainfall time is less than the ponding time; and $h_{0}$ denotes constant pressure head after the ponding time. Here, $q_{t}$ is assumed to be $K_{s 2} / 4$, and $h_{0}=0$. The initial condition is given by $h(z, t=0)=-z \times 10 m$.

The total simulation time and time step are taken to be $2 \mathrm{~h}$ and $0.01 \mathrm{~h}$, respectively. Let the number of nodes $N_{1}=64$, and $N_{2}=48$. Furthermore, NMG-MPPI(2) are applied to solve Eq. (30), where NMG with $\mu=10$ is adopted. The unit weight of the soil, the effective cohesion, and friction angle are $19.5 \mathrm{kN} / \mathrm{m}^{3}, 4.6 \mathrm{kPa}$, and $30^{\circ}$, respectively.

The numerical results indicate that the condition number of the coefficient matrix has dropped from $10^{6}$ to $10^{4}$. Fig. 16 shows that the pressure head increases with duration, while the pressure head at the interface has a greater increase. Besides, because the hydraulic conductivity of the bottom layer is lower than that of the upper layer, the lowest safety factor occurs at the interface (Fig. 17). Fig. 18 shows that $F_{S}$ varies with duration at the interface $(z=3 \mathrm{~m})$. After $t=1.88 \mathrm{~h}$, the slope may become unstable and cause landslides. Consequently, the results illustrate the soil layer structure will affect the distribution of the pressure head in the two-layer soil slopes during rainfall. When the hydraulic conductivity of the lower soil is lower than that of the upper one, it is easy to form ponding at the interface and induce landslides. 


\section{Summary and conclusions}

It is well-known that Picard iteration solves RE easier than Newton iteration without derivative computations. However, it is still time-consuming (Paniconi and Putti 1994; Lott et al. 2012; Zha et al. 2017). Firstly, a multi-step preprocessing algorithm is proposed to preprocess the system of linear equations. And the condition number of the coefficient matrix is greatly reduced, as shown in Figs. 5(a), 8 and 12. Furthermore, the multi-step preprocessing algorithm is combined with non-uniform multigrid. It is verified that the convergence rate of NMG-MPPI $(m)$ is faster than that of N-PI and N$\operatorname{MPPI}(m)$ in Fig. 4. The numerical results of infiltration in layered soils are shown in Figs. 9(a)-(c), 10(a)-(b), 13(a)-(b) and 14(a)-(b). The results demonstrate the effectiveness of NMG-MPPI $(m)$. The following conclusions can be drawn from numerical experiments and our analysis:

(1) We proposed an improved Picard iteration scheme with multistep preconditioner based on non-uniform multigrid correction V-Cycle scheme for simulating 1D unsaturated flow in porous media. Compared with conventional Picard iteration, NMG$\operatorname{MPPI}(m)$ has a faster convergence rate, higher calculation accuracy, as well as better calculation efficiency. The results also illustrate that the proposed NMG-MPPI $(m)$ exhibits good robustness for solving Richards' equation.

(2) The parameter $m$ in the proposed method (NMG-MPPI $(m)$ ) is suggested to be 2, which can ensure the calculation efficiency and improve the calculation accuracy. The parameter $\mu$ in the multigrid correction method should be selected reasonably 
according to the ill-condition of the system of linear equations. When the condition number of the matrix is large, the value of $\mu$ should increase accordingly.

(3) NMG-MPPI $(m)$ can investigate the 1D infiltration problem in the layered soil slopes effectively. If hydraulic conductivity of the lower soil is lower than that of the upper soil, the pressure head at the interface increases rapidly during rainfall and the minimum $F_{S}$ occurs at the interface of the layered soil slopes.

\section{Acknowledgements}

This work was supported by the National Key R\&D Program of China [no. 2018YFC1504702]; and the National Natural Science Foundation of China [no. 41672282].

\section{Data availability}

All data generated or analyzed during this study are included within the article.

\section{Conflict of interest}

The authors declare that they have no conflict of interest.

\section{References}

Abderahim, G., Mohammed, M., 2011. The use of a mixed scheme: mixed hybrid finite elements method/finite volumes (MHFE/FV), for the modeling of contaminants transport in unsaturated porous mediums. Arabian Journal of Geosciences, 4, 669680.

Benzi, M., 2002. Preconditioning techniques for large linear systems: a survey. Journal of Computational Physics, 182(2): 418-477.

Briggs, W. L., Henson, V. E., McCormick, S. F., 2000. A Multigrid Tutorial, 2nd ed., SIAM, Philadelphia, PA. 
Celia, M., Bouloutas, E., Zarba, R., 1990. A general mass-conservative numerical solution for the unsaturated flow equation. Water Resources Research. 26, 14831496.

Chávez-Negrete, C., F.J. Domínguez-Mota, D. Santana-Quinteros, 2018. Numerical solution of Richards equation of water flow by generalized finite differences. Computers and Geotechnics, 101, 168-175.

Cross, D., Onof, C., Winter, H. 2020. Ensemble estimation of future rainfall extremes with temperature dependent censored simulation. Advances in Water Resources, $136,103479$.

Gardner, W. R., 1958. Some steady-state solutions of the unsaturated moisture flow equation with application to evaporation from a water table. Soil Science, 85(4), 228-232.

Hashemi Jokar, M., Khosravi, A., Heidaripanah, A., \& Soltani, F. 2019. Unsaturated soils permeability estimation by adaptive neuro-fuzzy inference system. Soft Computing, 23, 6871-6881.

Hosseini, N., Bajalan, Z., Khoei, A. R. 2020. Numerical modeling of density-driven solute transport in fractured porous media with the extended finite element method. Advances in Water Resources, 136, 103453.

Illiano, D., Pop, I. S., Radu, F. A. 2020. Iterative schemes for surfactant transport in porous media. Computational Geosciences. https://doi.org/10.1007/s10596-02009949-2. 
Iverson, R.M., 2000. Landslide triggering by rain infiltration. Water Resources Research, 36(7), 1897-1910.

Jiang, S. H., Huang, J., 2016. Efficient slope reliability analysis at low-probability levels in spatially variable soils. Computers and Geotechnics, 75, 18-27.

Kuraz, M., Mayer, P., Pech, P., 2014. Solving the nonlinear Richards equation model with adaptive domain decomposition. Journal of Computational and Applied Mathematics, 270, 2-11.

List, F., Radu, F.A., 2016. A study on iterative methods for solving Richards' equation. Computational Geosciences, 20(2), 341-353.

Liu, C.Y., C.Y. Ku, C.C. Huang, 2015. Numerical Solutions for Groundwater Flow in Unsaturated Layered Soil with Extreme Physical Property Contrasts. International Journal of Nonlinear Sciences \& Numerical Simulation, 16(7), 325-335.

Lott, P. A., Walker, H. F., Woodward, C. S., Yang, U. M., 2012. An accelerated Picard method for nonlinear systems related to variably saturated flow. Advances in Water Resources, 38, 92-101.

Mishra, M., Gunturi, V. R., Maity, D. 2020. Teaching-learning-based optimisation algorithm and its application in capturing critical slip surface in slope stability analysis. Soft Computing, 24, 2969-2982.

Paniconi, C., M. Putti, 1994. A comparison of Picard and newton iteration in the numerical solution of multidimensional variably saturated flow problems. Water Resources Research, 30, 3357-3374.

Patankar, S. V., 1980. Numerical Heat Transfer and Fluid Flow, CRC press. 
Richards, L A., 1931. Capillary Conduction of Liquids Through Porous Mediums. Physics, 1(5), 318-333.

Sihag, P., Esmaeilbeiki, F., Singh, B., Ebtehaj, I., Bonakdari, H. 2019. Modeling unsaturated hydraulic conductivity by hybrid soft computing techniques. Soft Computing, 23, 12897-12910.

Šimůnek, J., Šejna, M., Saito, H., Sakai, M., van Genuchten, M.T., 2009. The HYDRUS-1D Software Package for Simulating the One-Dimensional Movement of Water, Heat, and Multiple Solutes in Variably-Saturated Media. Department of Environmental Sciences, University of California Riverside, Riverside, CA.

Srivastava, R., Yeh, T.C.J. 1991. Analytical solutions for one-dimensional, transient infiltration toward the water table in homogeneous layered soils. Water Resources Research. 27(5), 753-762.

Svyatskiy, D., Lipnikov, K., 2017. Second-order accurate finite volume schemes with the discrete maximum principle for solving Richards' equation on unstructured meshes. Advances in Water Resources, 104, 114-126.

Tracy, F.T., 2006. Clean two- and three-dimensional analytical solutions of Richards' equation for testing numerical solvers. Water Resources Research, 42(8), 8503, 111.

van Genuchten, M. T., 1980. A closed-form equation for predicting the hydraulic conductivity of unsaturated soils1. Soil Science Society of America Journal, 44(5), 892-898.

Wang, H.F., Anderson, M.P., 1982. Introduction to groundwater modeling. Freeman. 
Wang, K., Zhang, J. 2003. MSP: A Class of Parallel Multistep Successive Sparse Approximate Inverse Preconditioning Strategies. SIAM Journal on Scientific Computing, 24(4), 1141-1156.

Wu, L.Z., G.G. Liu, L.C. Wang, L.M. Zhang, B.E. Li, B. Li, 2016. Numerical analysis of 1D coupled infiltration and deformation in layered unsaturated porous medium. Environmental Earth Sciences. 75(9), 761, 1-11.

Wu, L.Z., Jinsong, Huang, W. Fan, X. Li, 2020a. Hydro-mechanical coupling in unsaturated soils covering a non-deformable structure. Computers and Geotechnics. 117, 103287.

Wu, L.Z., S.R. Zhu, J.B. Peng, 2020b. Application of the Chebyshev spectral method to the simulation of groundwater flow and rainfall-induced landslides. Applied Mathematical Modelling. 80, 408-425.

Wu, L.Z., Y. Zhou, P. Sun, J.S. Shi, G.G. Liu, L.Y. Bai, 2017. Laboratory characterization of rainfall-induced loess slope failure. Catena, 150, 1-8.

Zeng, J.C., Y.Y. Zha, J.Z. Yang, 2018. Switching the Richards' equation for modeling soil water movement under unfavorable conditions. Journal of Hydrology, 563, 942-949.

Zha, Y., Yang, J., Shi, L., Song, X., 2013. Simulating One-Dimensional Unsaturated Flow in Heterogeneous Soils with Water Content-Based Richards Equation. Vadose Zone Journal, 12(2), 1-13. 
Zha, Y., Yang, J., Yin, L., Zhang, Y., Zeng, W., Shi, L. 2017. A modified Picard iteration scheme for overcoming numerical difficulties of simulating infiltration into dry soil. Journal of Hydrology, 551, 56-69.

Zhang, Z., Wang, W., Yeh, T. J., Chen, L., Wang, Z., Duan, L., An, K., Gong, C., 2016. Finite analytic method based on mixed-form Richards' equation for simulating water flow in vadose zone. Journal of Hydrology, 537, 146-156.

Zhou, X., Huang, W., Liu, Z., Chen, H. 2018. Assessment of slope stability under uncertain circumstances. Soft Computing, 22, 5735-5745.

Zhu, S.R., Wu, L.Z., Peng, J.B., 2020. An improved Chebyshev semi-iterative method for simulating rainfall infiltration in unsaturated soils and its application to shallow landslides, Journal of Hydrology, 590, 125157. 


\section{Figure and Table Captions}

Table 1. Numerical results for Test 1.

Table 2 Hydraulic conductivity for different cases in two-layer soils.

Table 3 Hydraulic conductivity for different cases in three-layer soils.

Table 4. Parameter of van Genuchten model in application examples.

Figure 1. Schematic drawing of control volume method based on non-uniform grid nodes and 1D infiltration model for two-layer soils.

Figure 2. Schematic drawing of non-uniform multigrid correction V-Cycle scheme.

Figure 3. Brief flowchart of the proposed NMG-MPPI $(m)$.

Figure 4. Comparison of convergence rates for the proposed schemes under different number of steps.

Figure 5. Comparison of results for Test 1 over different number of steps using the NMG-MPPI $(m)$ with $\mu=3$ : (a) condition number; (b) maximum relative error (\%).

Figure 6. Comparison of the numerical solutions obtained using NMG-MPPI(2) with analytical solutions for Test 1.

Figure 7. Comparison of the speed-up ratios at different time steps and number of nodes:

(a) $S_{N-P I / N-M P P I(1)} ;$ (b) $S_{N-P I / N M G-M P P I(2)}$.

Figure 8. Condition number for Cases 1 to 8.

Figure 9. Numerical solutions obtained using the NMG-MPPI(2): (a) Case 3; (b) Case 5; (c) Case 7.

Figure 10. Numerical results for Cases 1 to 8: (a) Iterations; (b) Speed-up ratio.

Figure 11. 1D infiltration model for three-layer soils. 
Figure 12. Condition number for Cases 9 to 13.

Figure 13. Numerical solutions obtained using the NMG-MPPI(2): (a) Case 10; (b) Case 12.

Figure 14. Numerical results for Cases 9 to 13: (a) Iterations; (b) Speed-up ratio.

Figure 15. Schematic diagram for two-layer soil slopes.

Figure 16. Computed profile of pressure head for application examples.

Figure 17. Computed profile of $F_{S}$ for application examples.

Figure 18. Results of $F_{S}$ at the interface $(z=3 \mathrm{~m})$ for application examples. 
Table 1. Numerical results for Test 1.

\begin{tabular}{ccccccccccc}
\hline \multicolumn{2}{c}{ Conditions } & \multicolumn{3}{c}{$\begin{array}{c}\text { Average iterations for each } \\
\text { time step }\end{array}$} & \multicolumn{2}{c}{ Running time (s) } & \multicolumn{2}{c}{ Maximum relative error (\%) } \\
\hline $\begin{array}{c}\text { Number } \\
\text { of nodes }\end{array}$ & $\Delta t$ & N-PI & $\begin{array}{c}\text { N- } \\
\text { MPPI(1) }\end{array}$ & $\begin{array}{c}\text { NMG- } \\
\text { MPPI(2) }\end{array}$ & N-PI & $\begin{array}{c}\text { N- } \\
\text { MPPI(1) }\end{array}$ & $\begin{array}{c}\text { NMG- } \\
\text { MPPI(2) }\end{array}$ & N-PI & $\begin{array}{c}\text { N- } \\
\text { MPPI(1) }\end{array}$ & $\begin{array}{c}\text { NMG- } \\
\text { MPPI(2) }\end{array}$ \\
\hline 97 & $0.1 \mathrm{~h}$ & 386 & 75 & 4 & 29.02 & 15.95 & 7.65 & 1.3979 & 1.3979 & 1.1353 \\
& $0.05 \mathrm{~h}$ & 275 & 55 & 4 & 41.94 & 23.22 & 14.07 & 0.7091 & 0.7091 & 0.6073 \\
& $0.025 \mathrm{~h}$ & 198 & 40 & 3 & 61.52 & 34.77 & 21.48 & 0.3622 & 0.3622 & 0.3439 \\
129 & $0.1 \mathrm{~h}$ & 663 & 125 & 5 & 82.72 & 43.70 & 16.10 & 1.3931 & 1.3931 & 1.0706 \\
& $0.05 \mathrm{~h}$ & 473 & 91 & 5 & 119.32 & 63.42 & 28.51 & 0.7017 & 0.7017 & 0.4547 \\
& $0.025 \mathrm{~h}$ & 339 & 66 & 4 & 178.88 & 92.79 & 46.26 & 0.3556 & 0.3556 & 0.2353 \\
161 & $0.1 \mathrm{~h}$ & 1009 & 189 & 7 & 198.72 & 99.56 & 30.57 & 1.3896 & 1.3896 & 1.1834 \\
& $0.05 \mathrm{~h}$ & 720 & 136 & 6 & 281.36 & 147.03 & 52.92 & 0.6994 & 0.6994 & 0.4530 \\
& $0.025 \mathrm{~h}$ & 516 & 98 & 5 & 410.99 & 214.75 & 88.91 & 0.3525 & 0.3525 & 0.1635 \\
\hline
\end{tabular}


Table 2 Hydraulic conductivity for different cases in two-layer soils.

\begin{tabular}{ccccccccc}
\hline Case number & 1 & 2 & 3 & 4 & 5 & 6 & 7 & 8 \\
\hline$K_{s 1}(\mathrm{~m} / \mathrm{s})$ & $10^{-1}$ & $10^{-1}$ & $10^{-1}$ & $10^{-1}$ & $10^{-1}$ & $10^{-1}$ & $10^{-1}$ & $10^{-1}$ \\
$K_{s 2}(\mathrm{~m} / \mathrm{s})$ & $10^{-2}$ & $10^{-3}$ & $10^{-4}$ & $10^{-5}$ & $10^{-6}$ & $10^{-7}$ & $10^{-8}$ & $10^{-9}$ \\
\hline
\end{tabular}

Table 3 Hydraulic conductivity for different cases in three-layer soils.

\begin{tabular}{cccccc}
\hline Case number & 9 & 10 & 11 & 12 & 13 \\
\hline$K_{s 1}(\mathrm{~m} / \mathrm{s})$ & 1 & 1 & 1 & 1 & 1 \\
$K_{s 2}(\mathrm{~m} / \mathrm{s})$ & $10^{-3}$ & $10^{-4}$ & $10^{-5}$ & $10^{-6}$ & $10^{-7}$ \\
$K_{s 3}(\mathrm{~m} / \mathrm{s})$ & 1 & 1 & 1 & 1 & 1 \\
\hline
\end{tabular}

Table 4. Parameter of van Genuchten model in application examples.

\begin{tabular}{cccccc}
\hline Soil texture & $K_{s}(\mathrm{~m} / \mathrm{s})$ & $\theta_{s}$ & $\theta_{r}$ & $\beta(1 / \mathrm{m})$ & $n$ \\
\hline $\begin{array}{c}\text { Glendale clay loam } \\
\text { Berino loamy fine } \\
\text { sand }\end{array}$ & $1.5162 \times 10^{-6}$ & 0.4686 & 0.106 & 1.04 & 1.3954 \\
& $6.2616 \times 10^{-5}$ & 0.3658 & 0.0286 & 2.8 & 2.239 \\
\hline
\end{tabular}




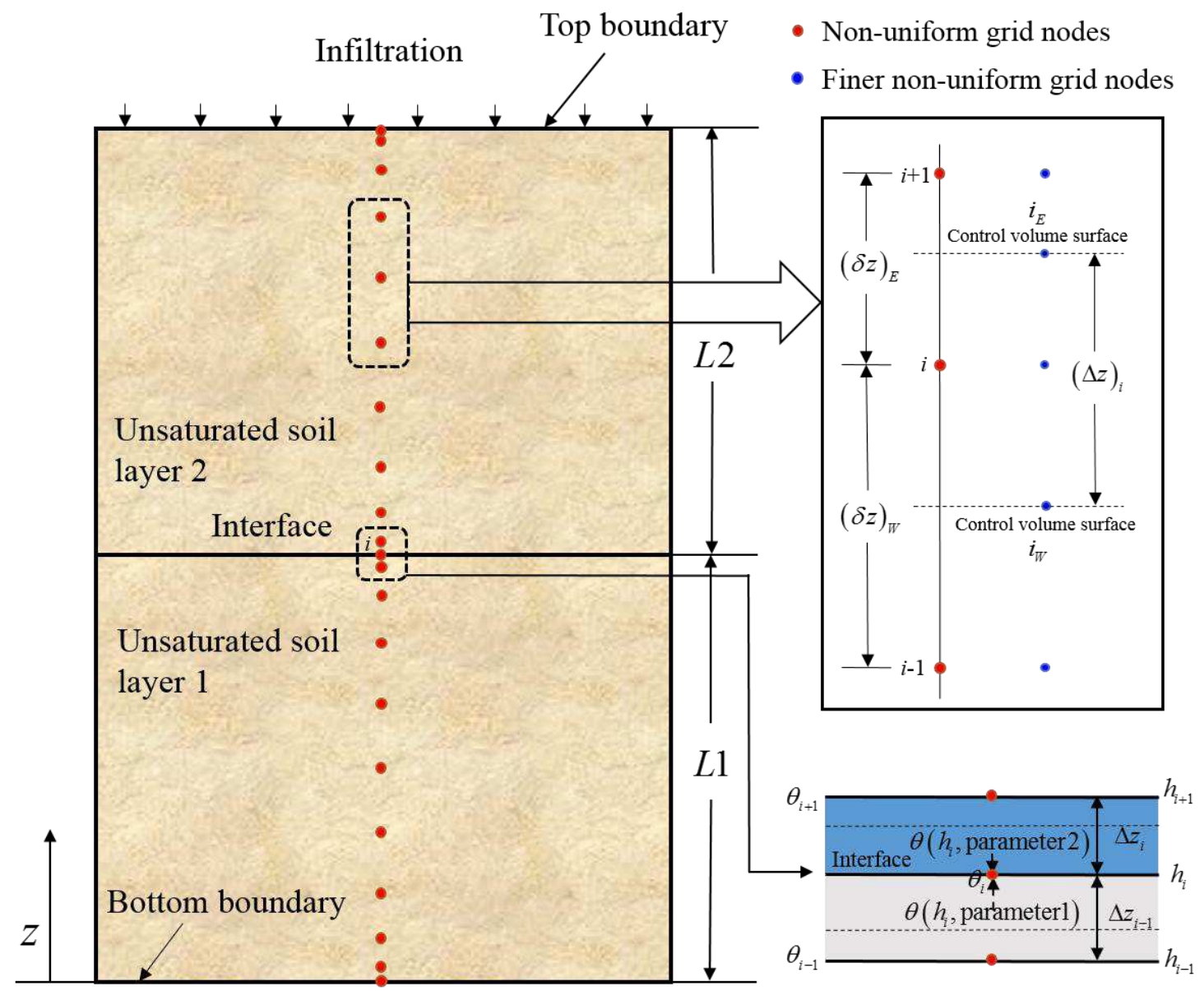

Figure 1. Schematic drawing of control volume method based on non-uniform grid nodes and 1D infiltration model for two-layer soils. 


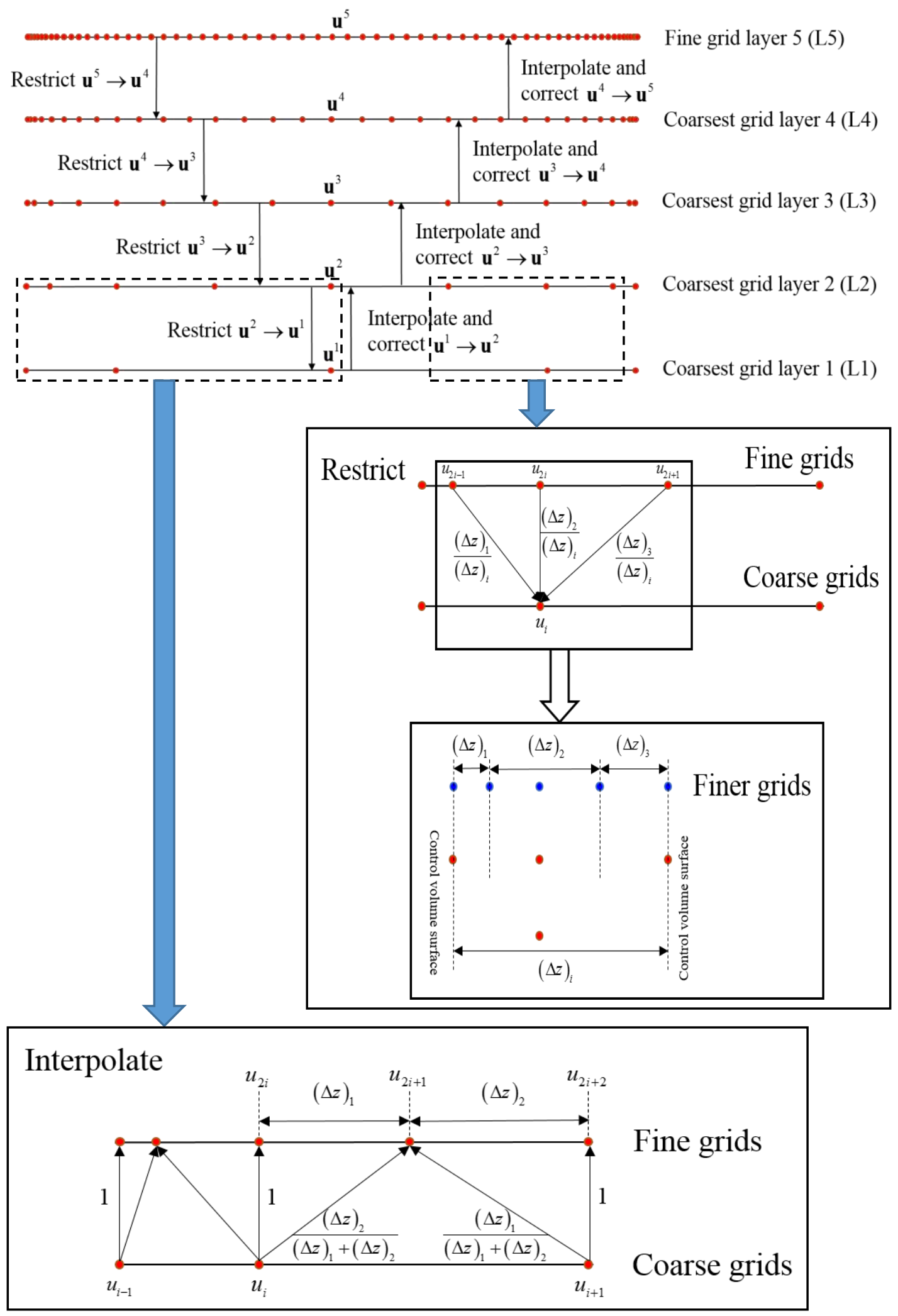

Figure 2. Schematic drawing of non-uniform multigrid correction V-Cycle scheme. 


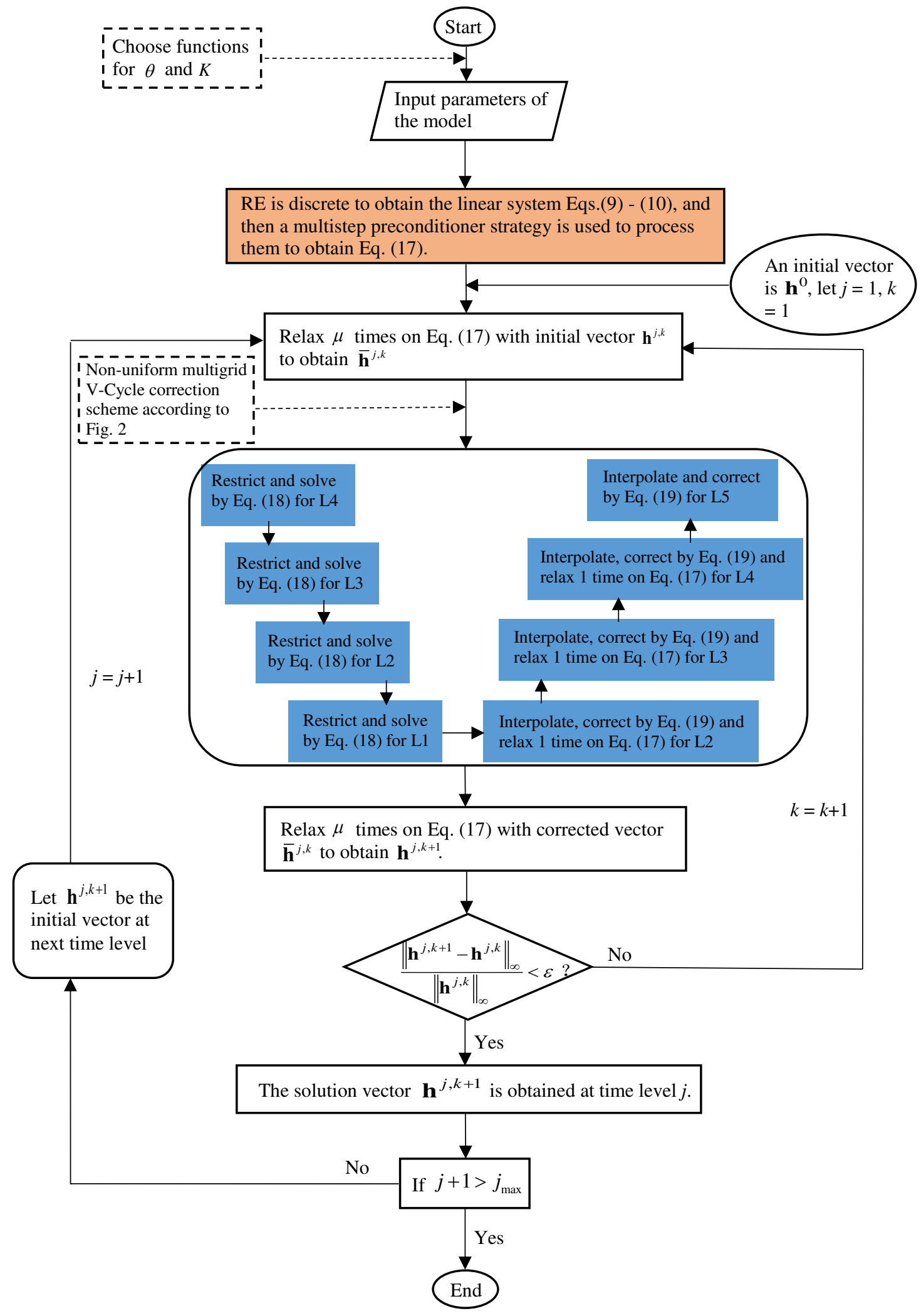

Figure 3. Brief flowchart of the proposed NMG-MPPI $(m)$. 


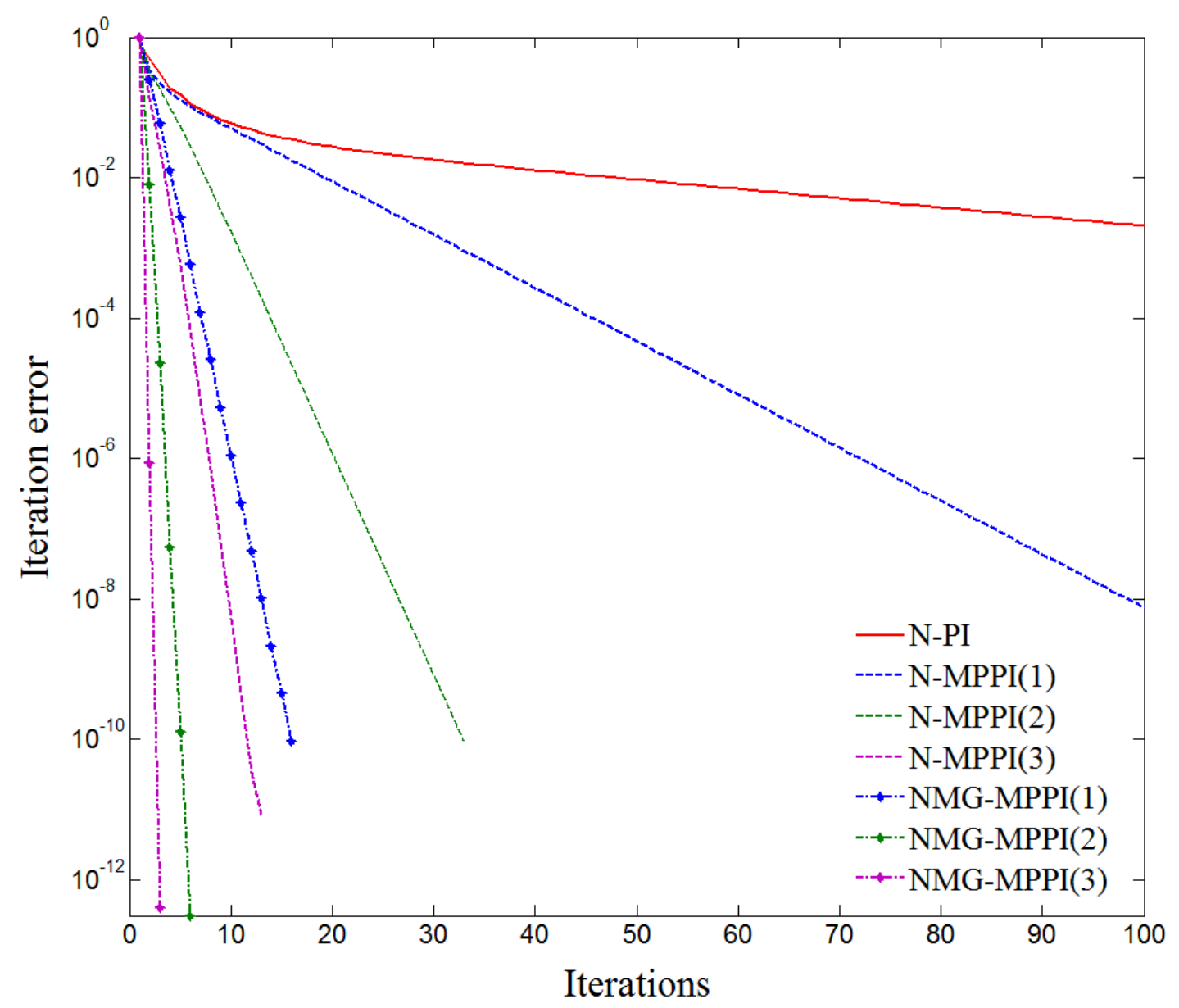

Figure 4. Comparison of convergence rates for the proposed schemes under different number of steps. 

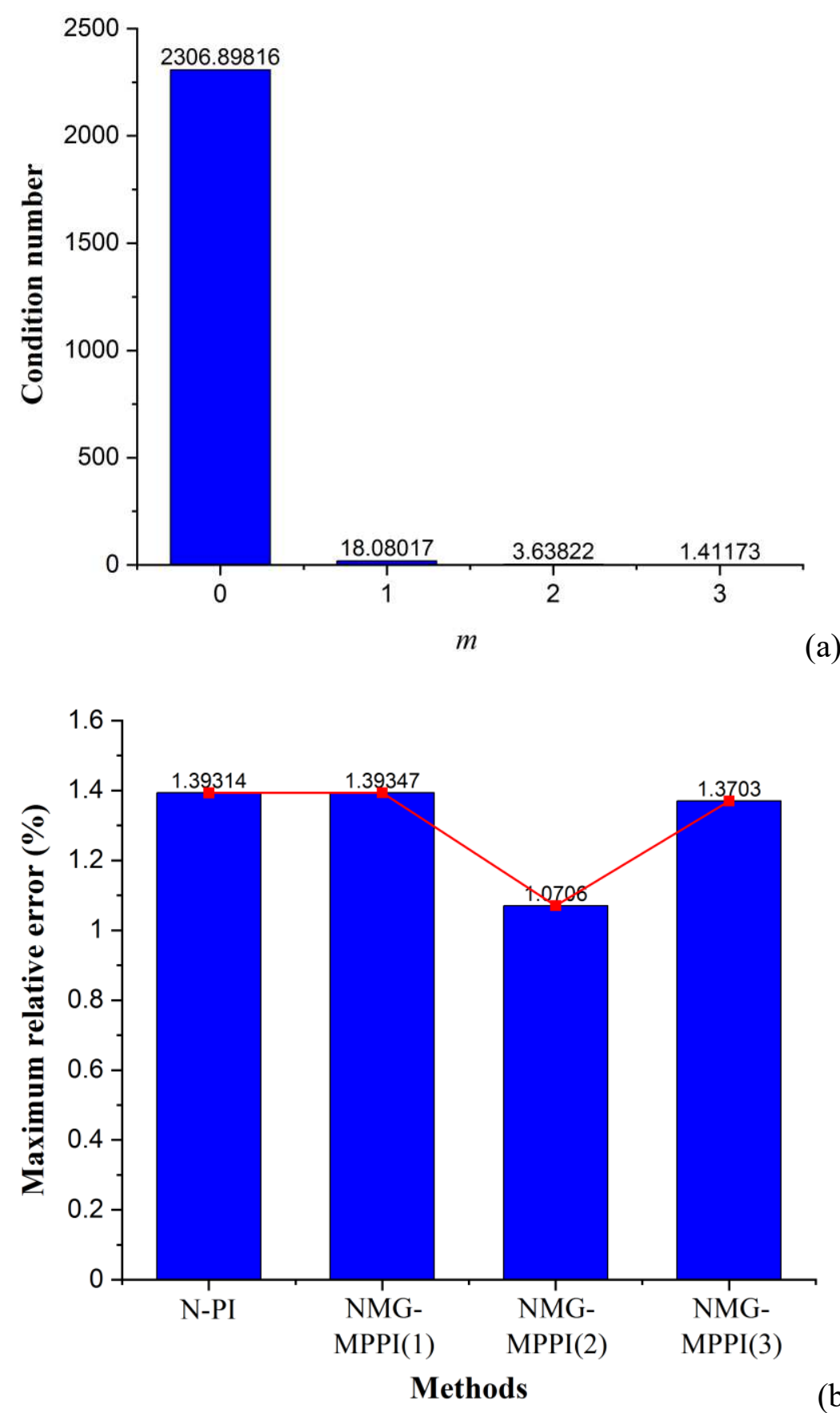

Figure 5. Comparison of results for Test 1 over different number of steps using the $\operatorname{NMG}-\operatorname{MPPI}(m)$ with $\mu=3$ : (a) condition number; (b) maximum relative error $(\%)$. 

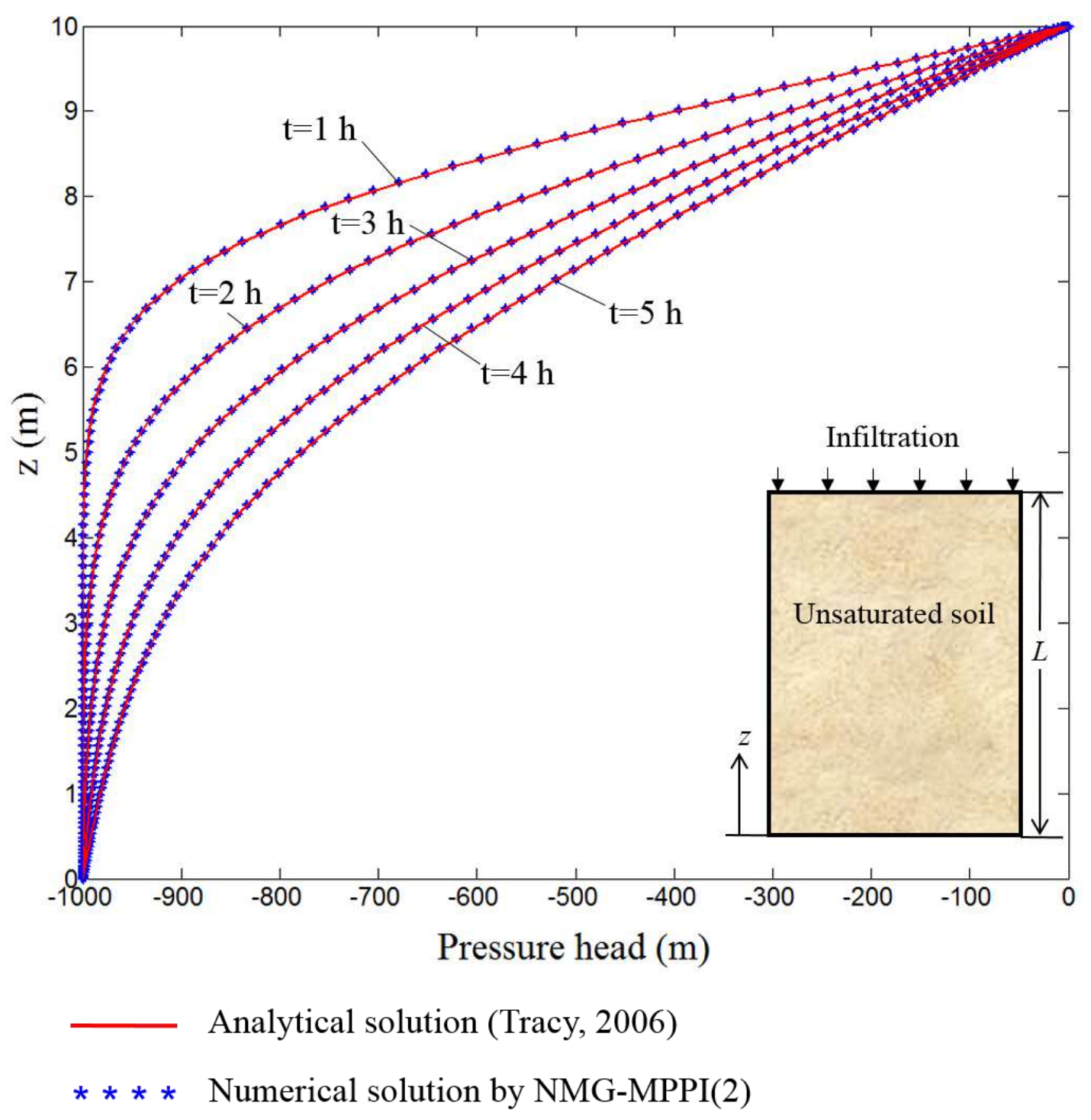

Figure 6. Comparison of the numerical solutions obtained using NMG-MPPI(2) with analytical solutions for Test 1. 

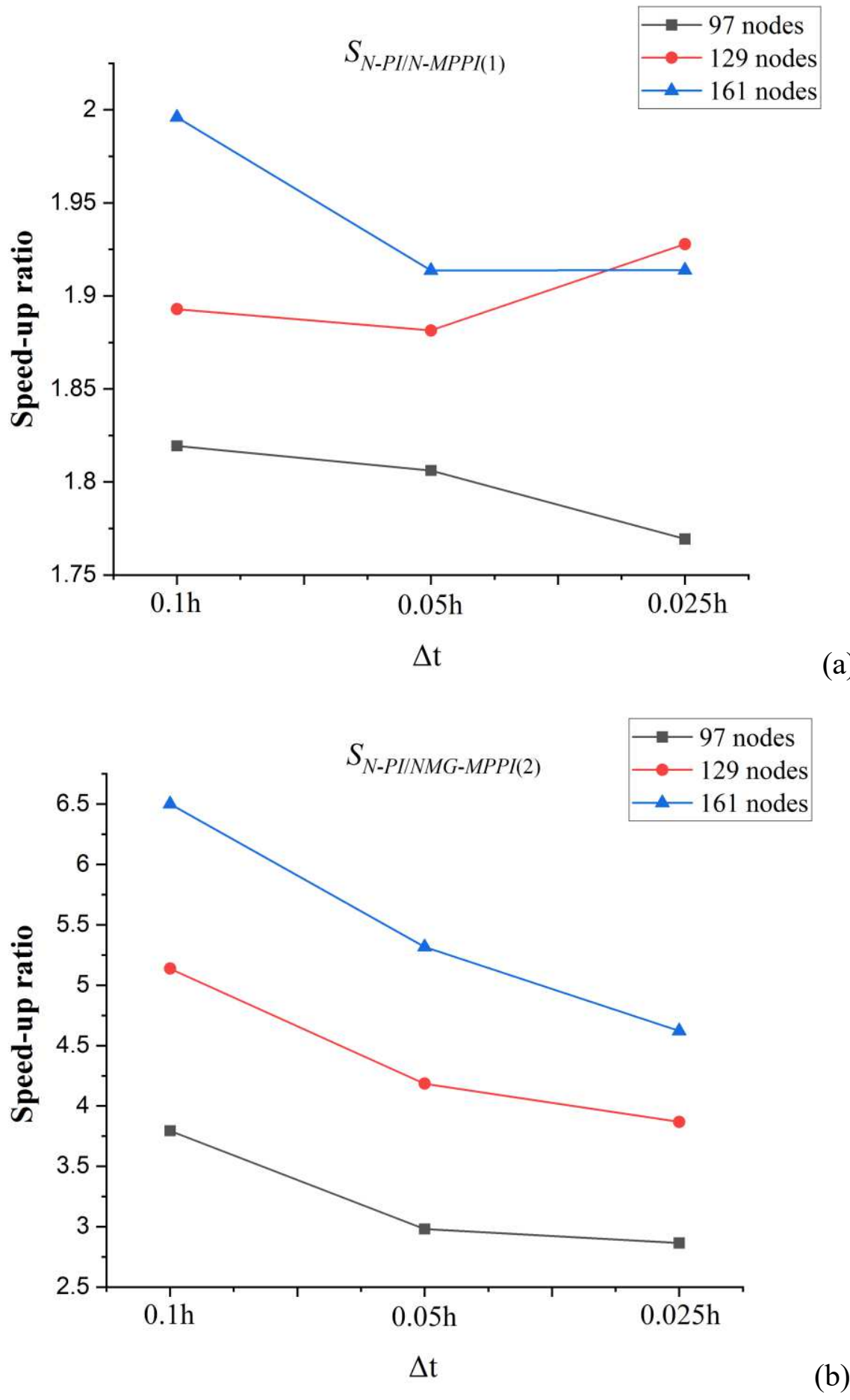

Figure 7. Comparison of the speed-up ratios at different time steps and number of nodes:

(a) $S_{N-P I / N-M P P I(1)}$; (b) $S_{N-P I / N M G-M P P I(2)}$. 


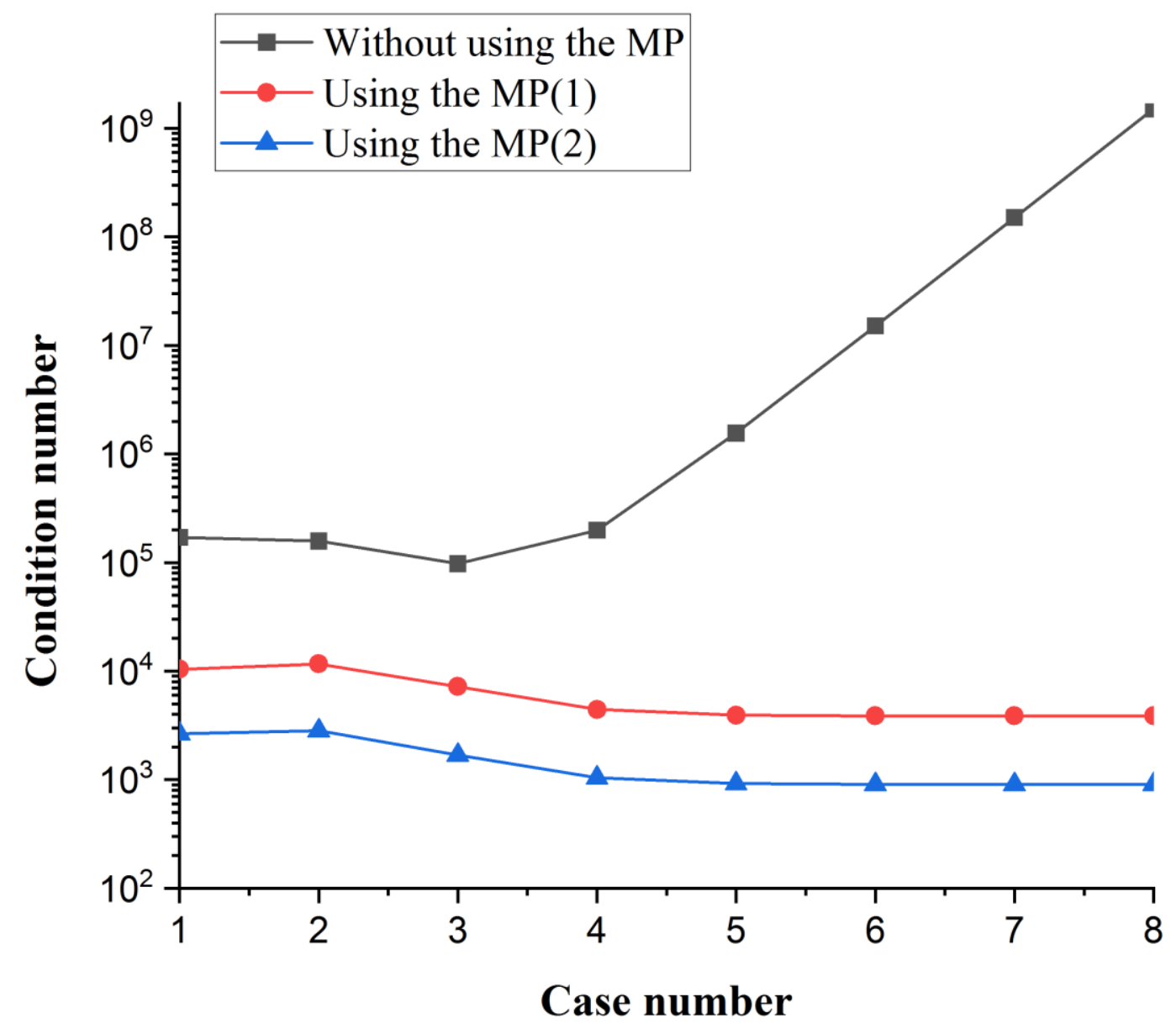

Figure 8. Condition number for Cases 1 to 8 . 


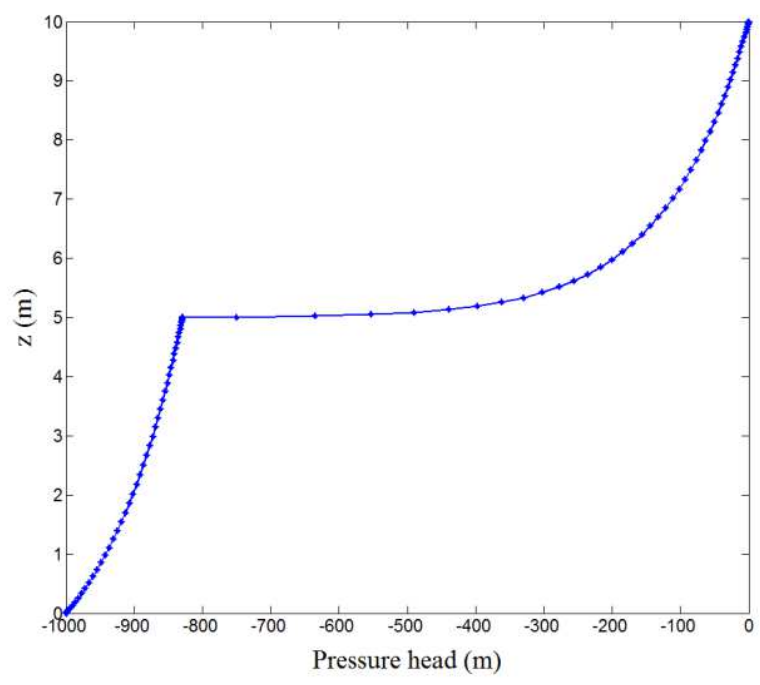

(a)

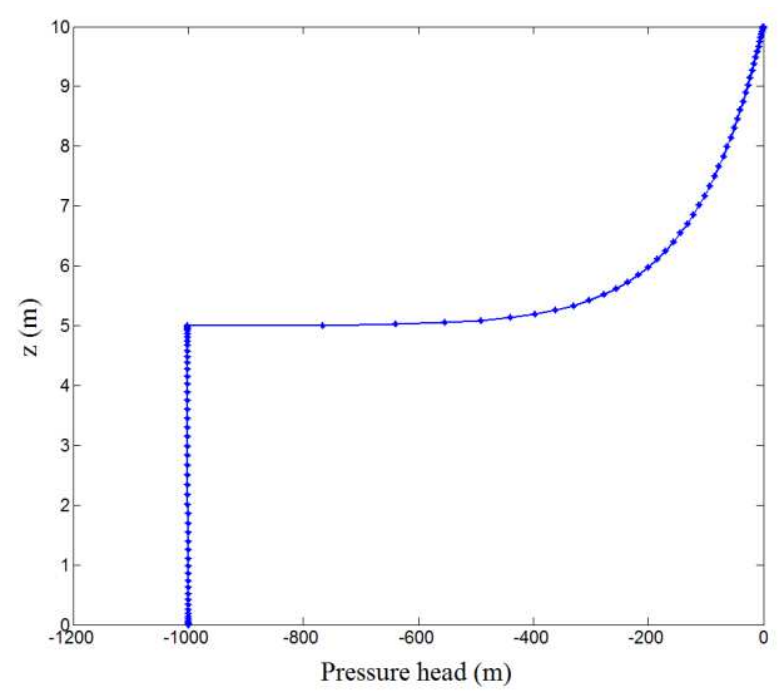

(b)

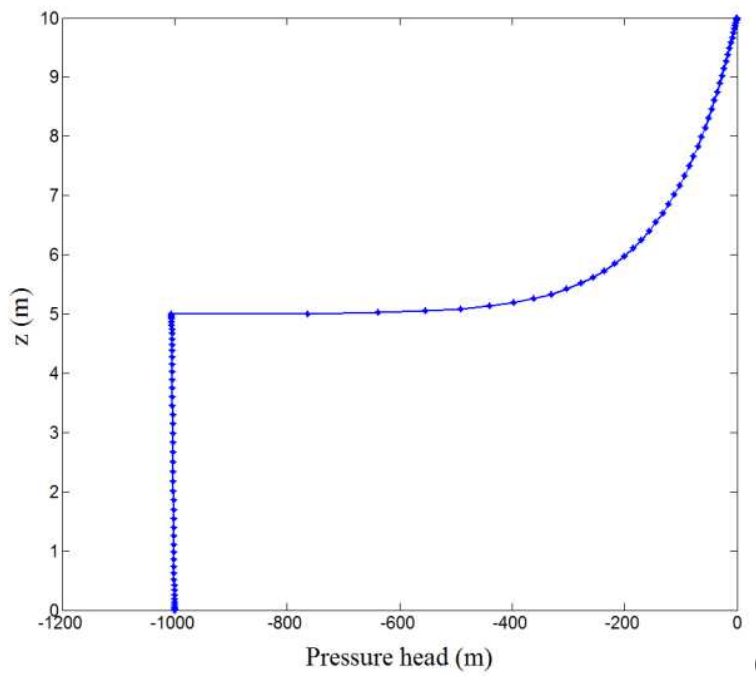

(c)

Figure 9. Numerical solutions obtained using the NMG-MPPI(2): (a) Case 3; (b) Case 5; (c) Case 7. 


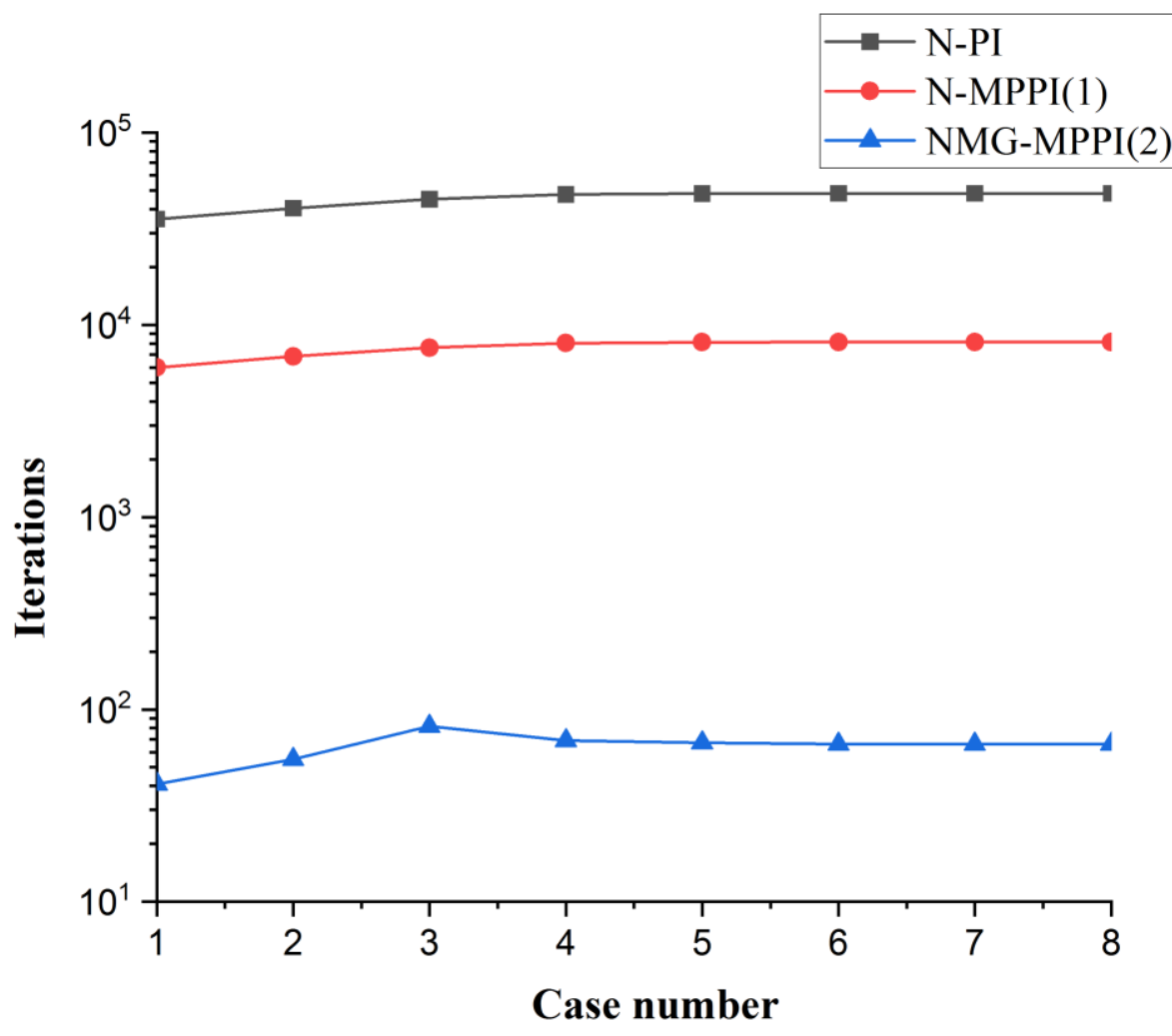

(a)

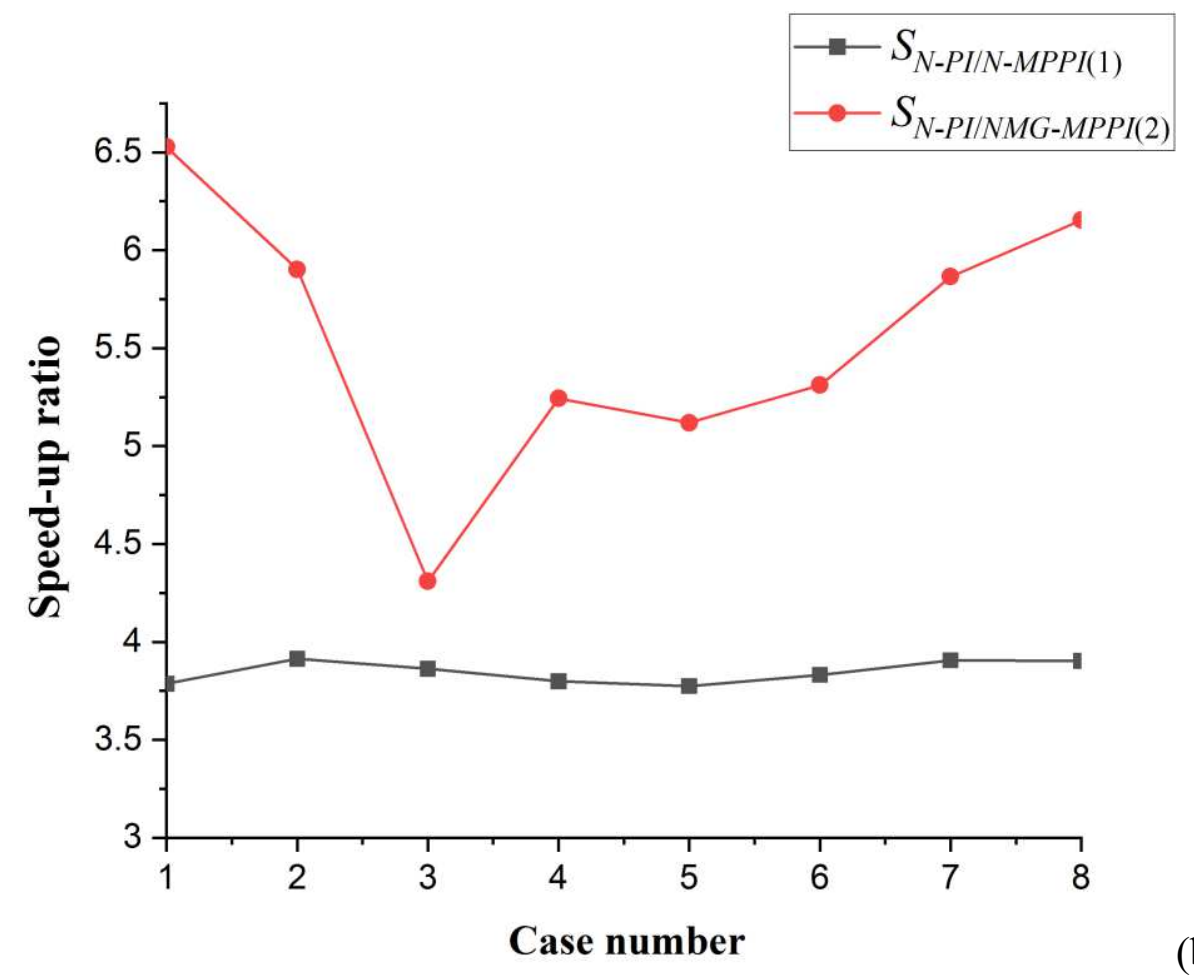

Figure 10. Numerical results for Cases 1 to 8: (a) Iterations; (b) Speed-up ratio. 


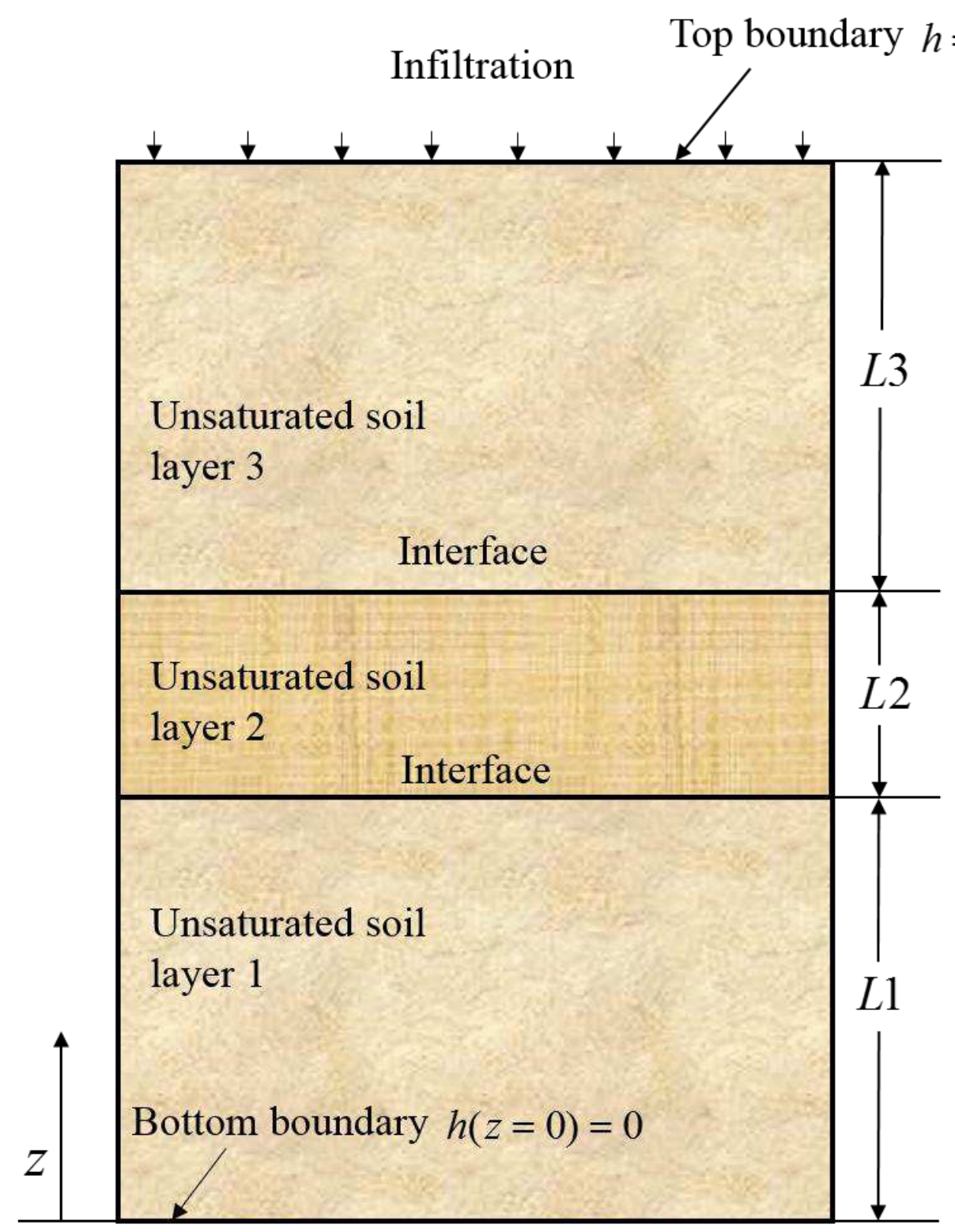

Figure 11. 1D infiltration model for three-layer soils. 


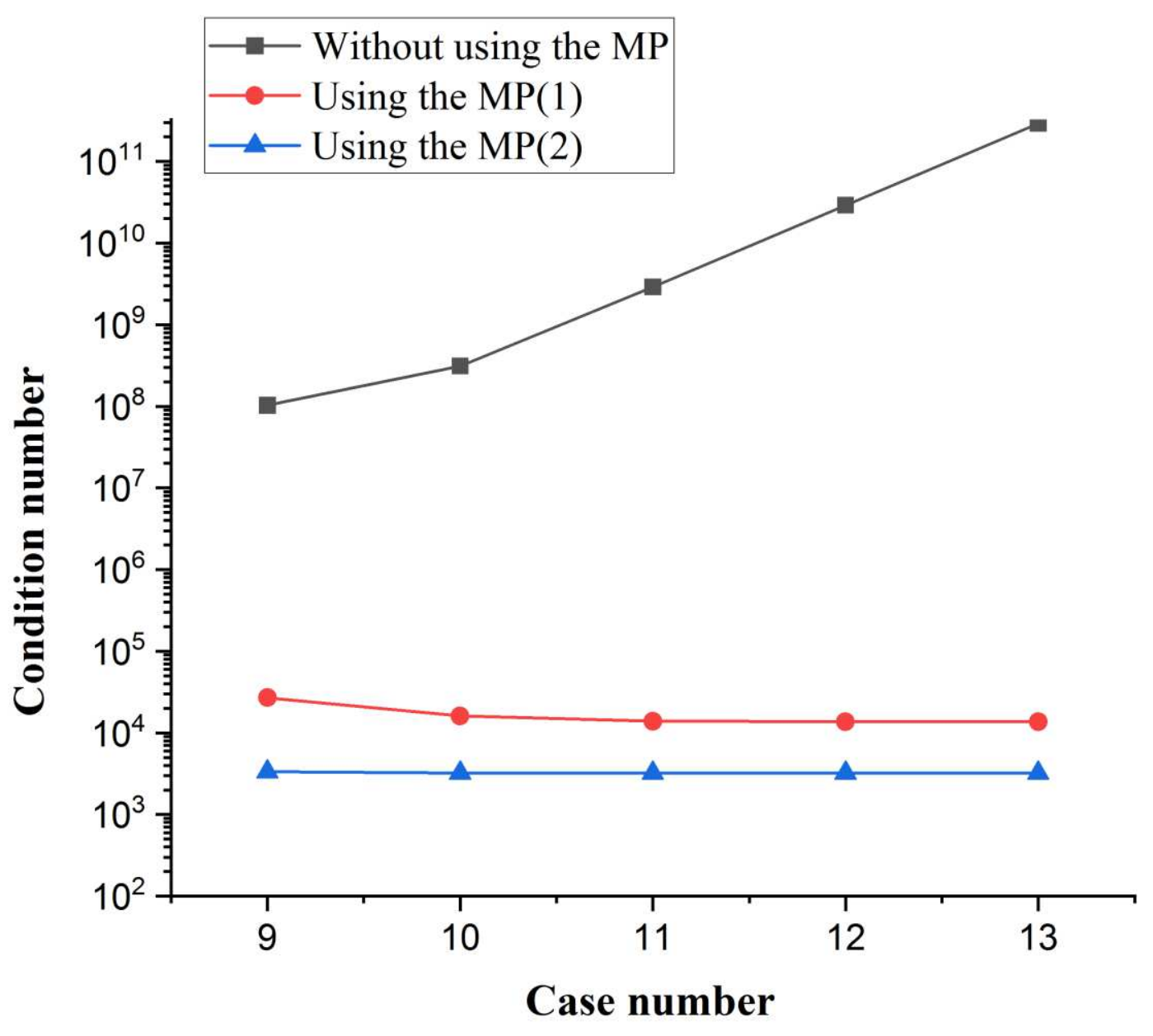

Figure 12. Condition number for Cases 9 to 13. 

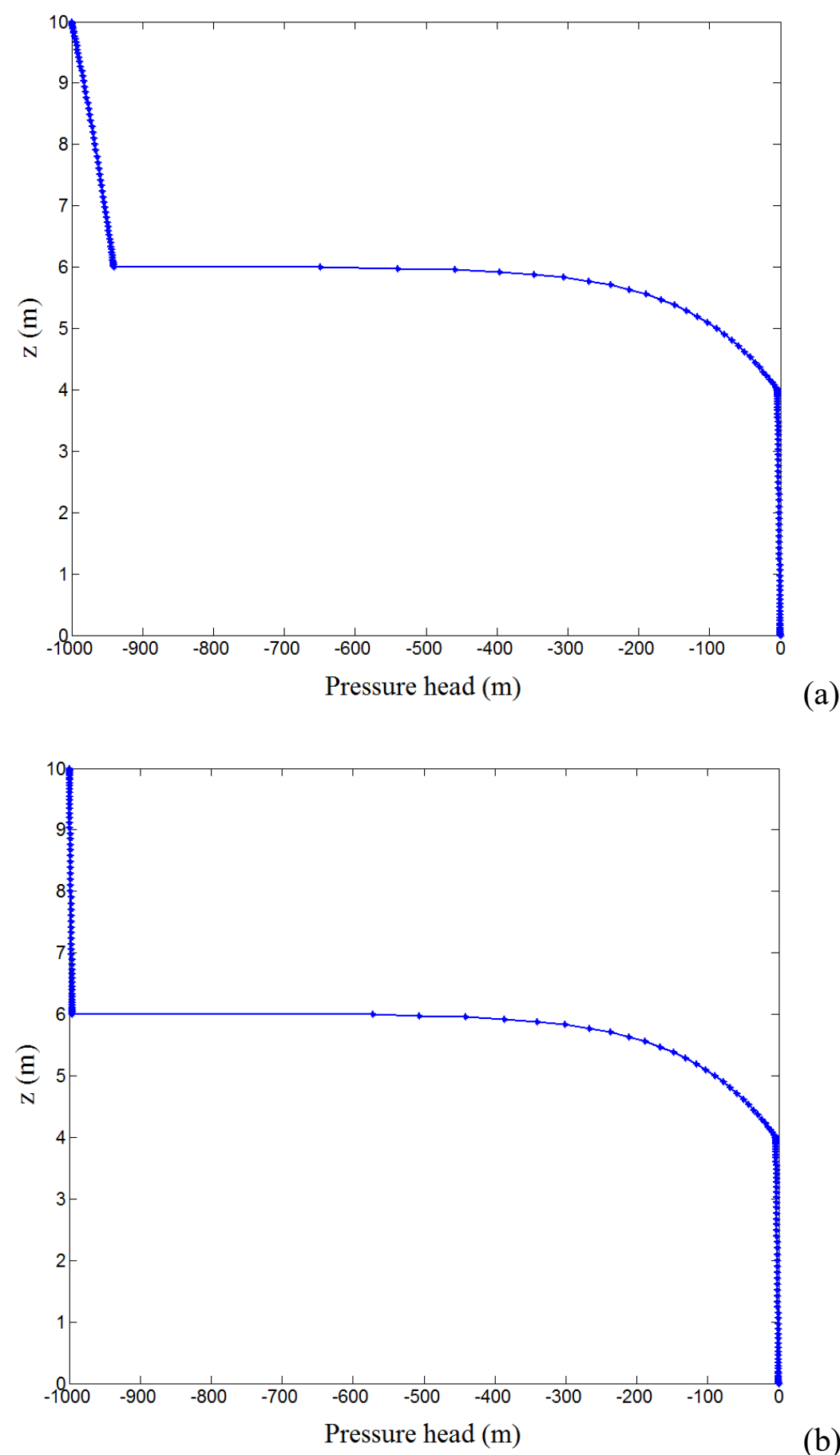

(b)

Figure 13. Numerical solutions obtained using the NMG-MPPI(2): (a) Case 10; (b) Case 12. 

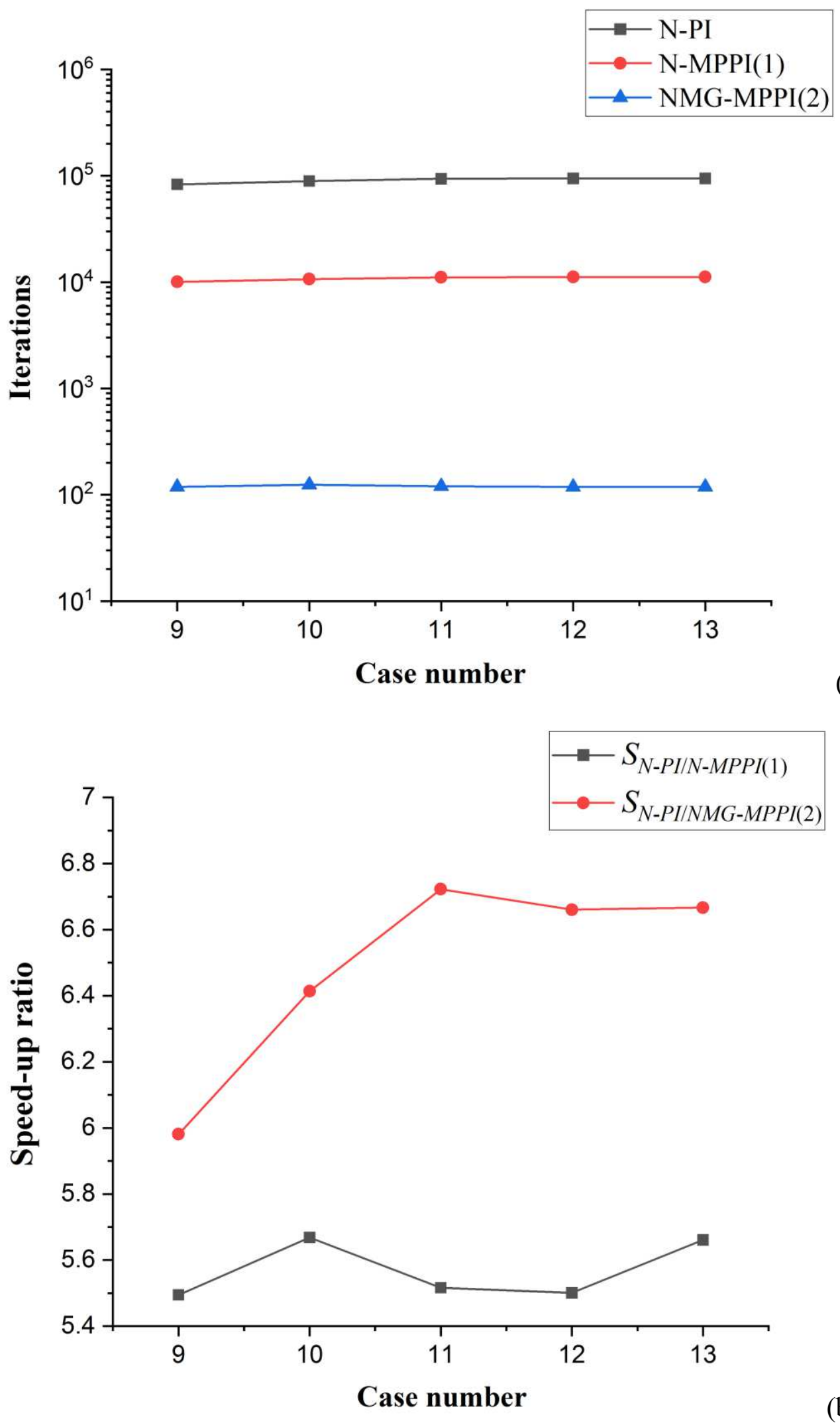

(a)

(b)

Figure 14. Numerical results for Cases 9 to 13: (a) Iterations; (b) Speed-up ratio. 


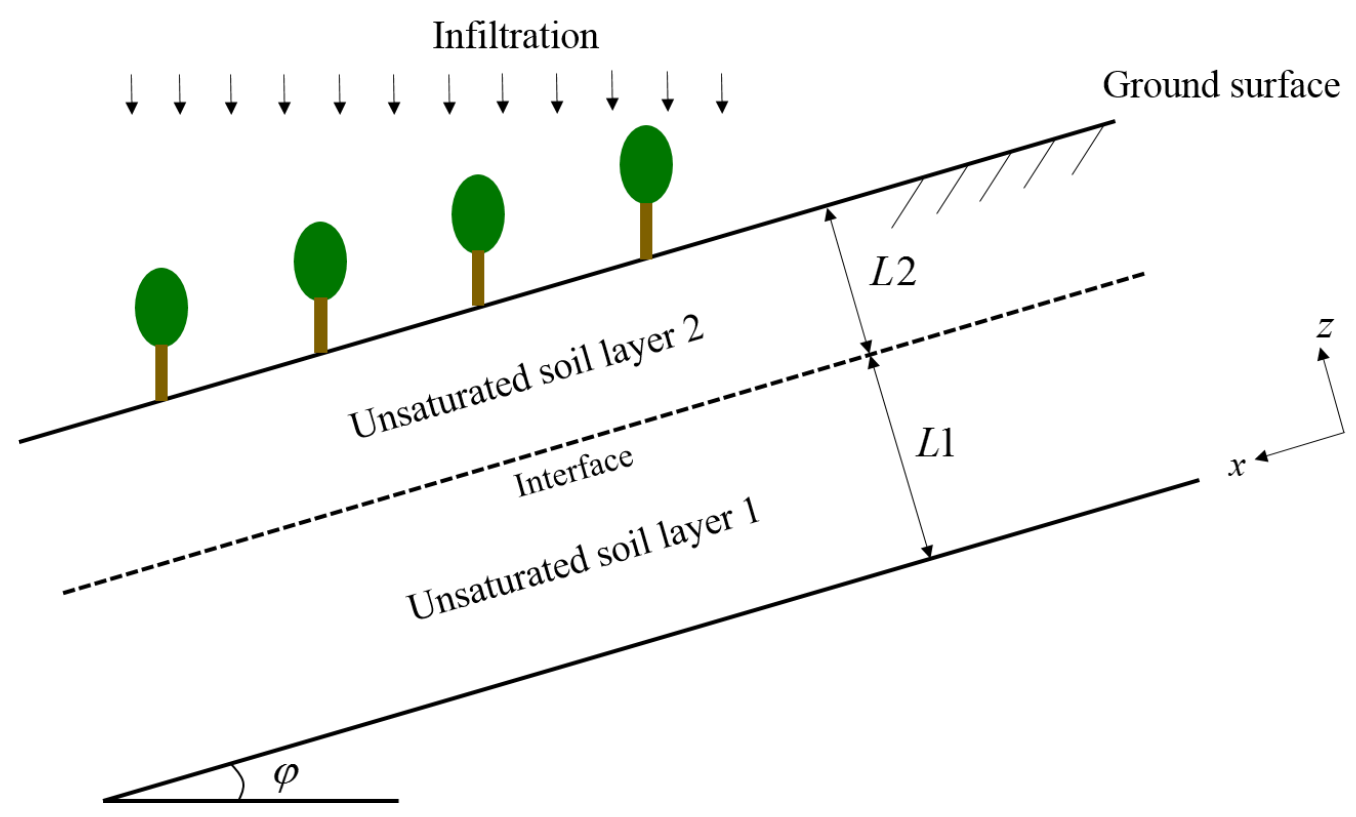

Figure 15. Schematic diagram for two-layer soil slopes. 


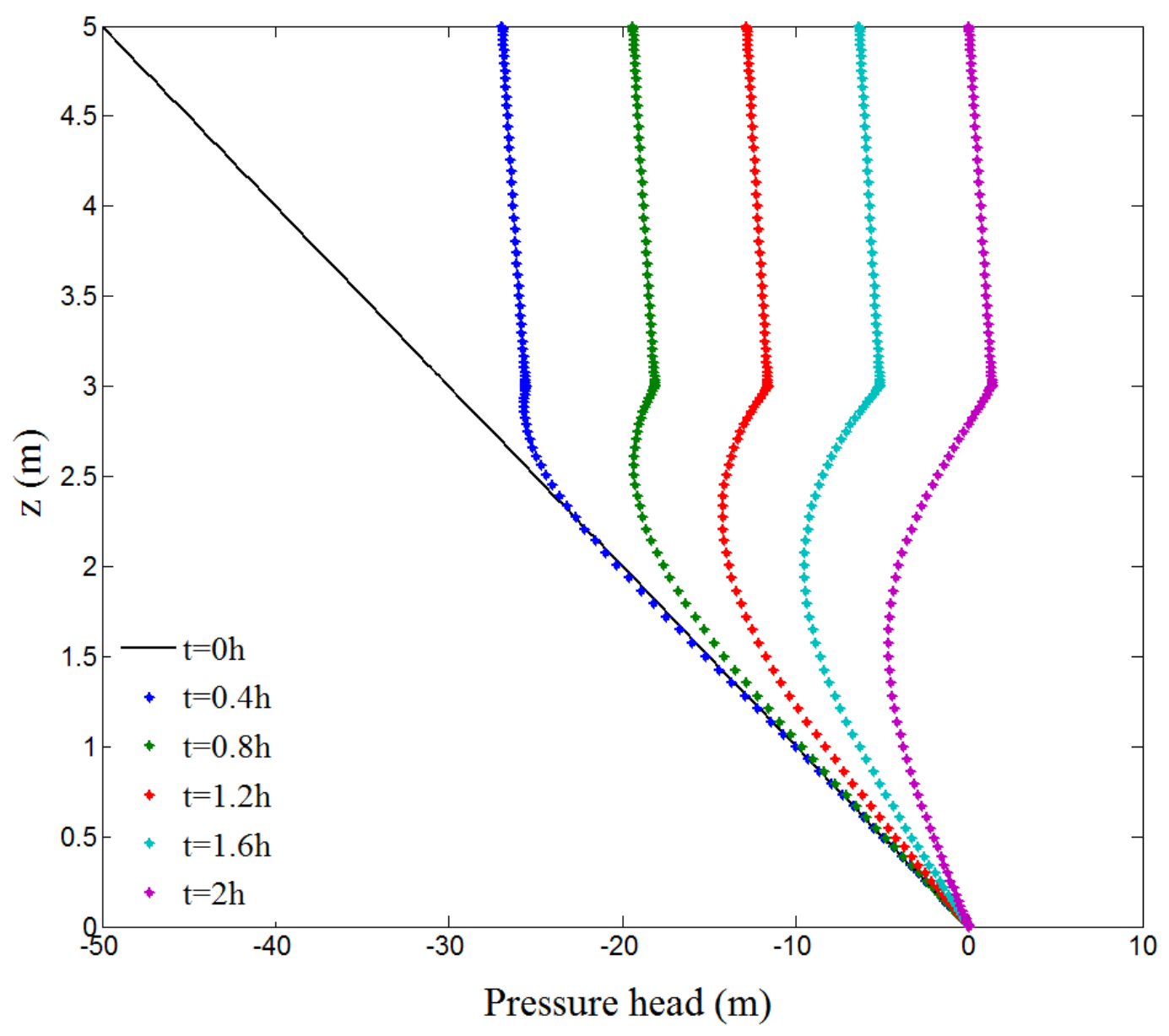

Figure 16. Computed profile of pressure head for application examples. 


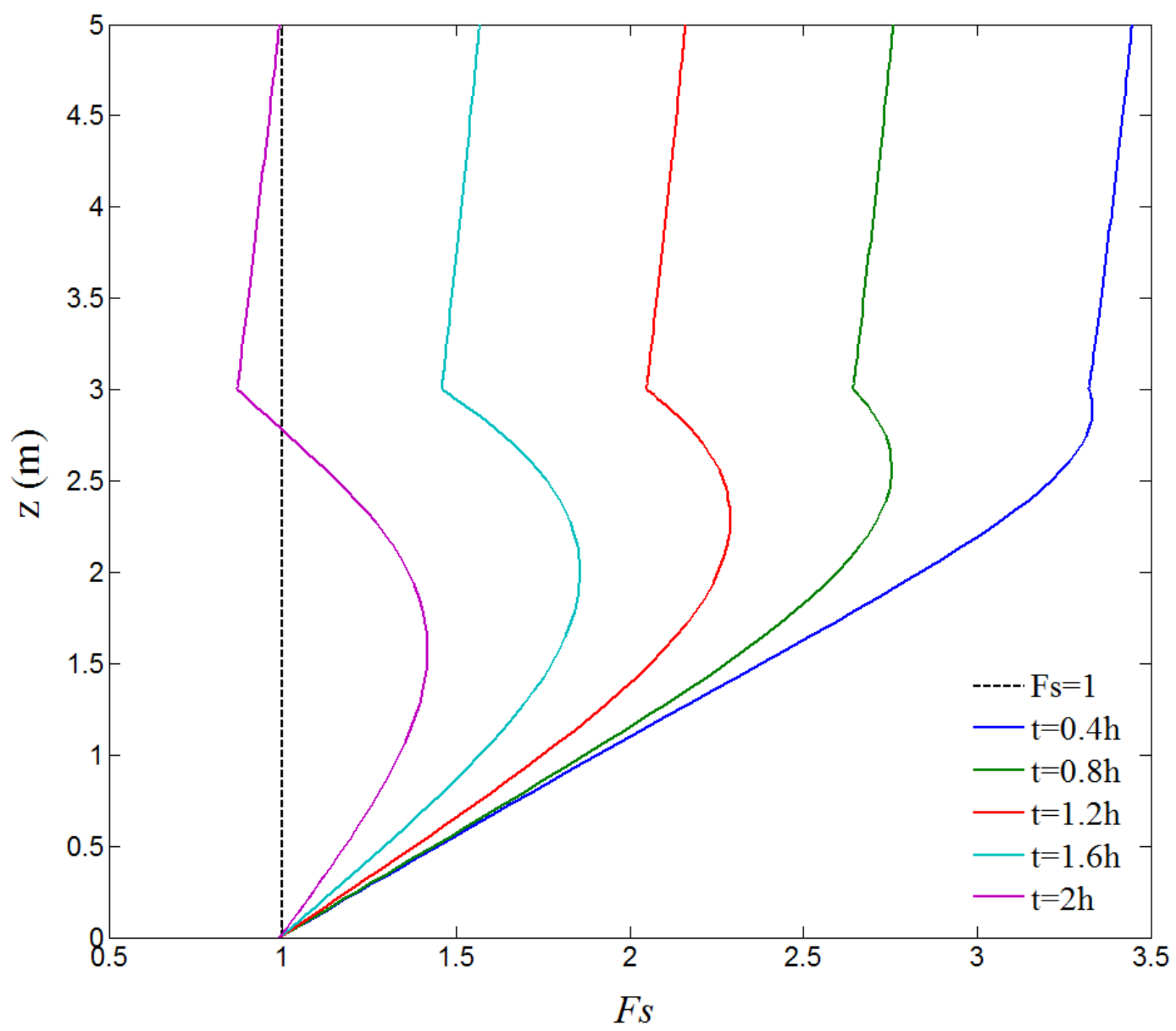

Figure 17. Computed profile of $F_{S}$ for application examples. 


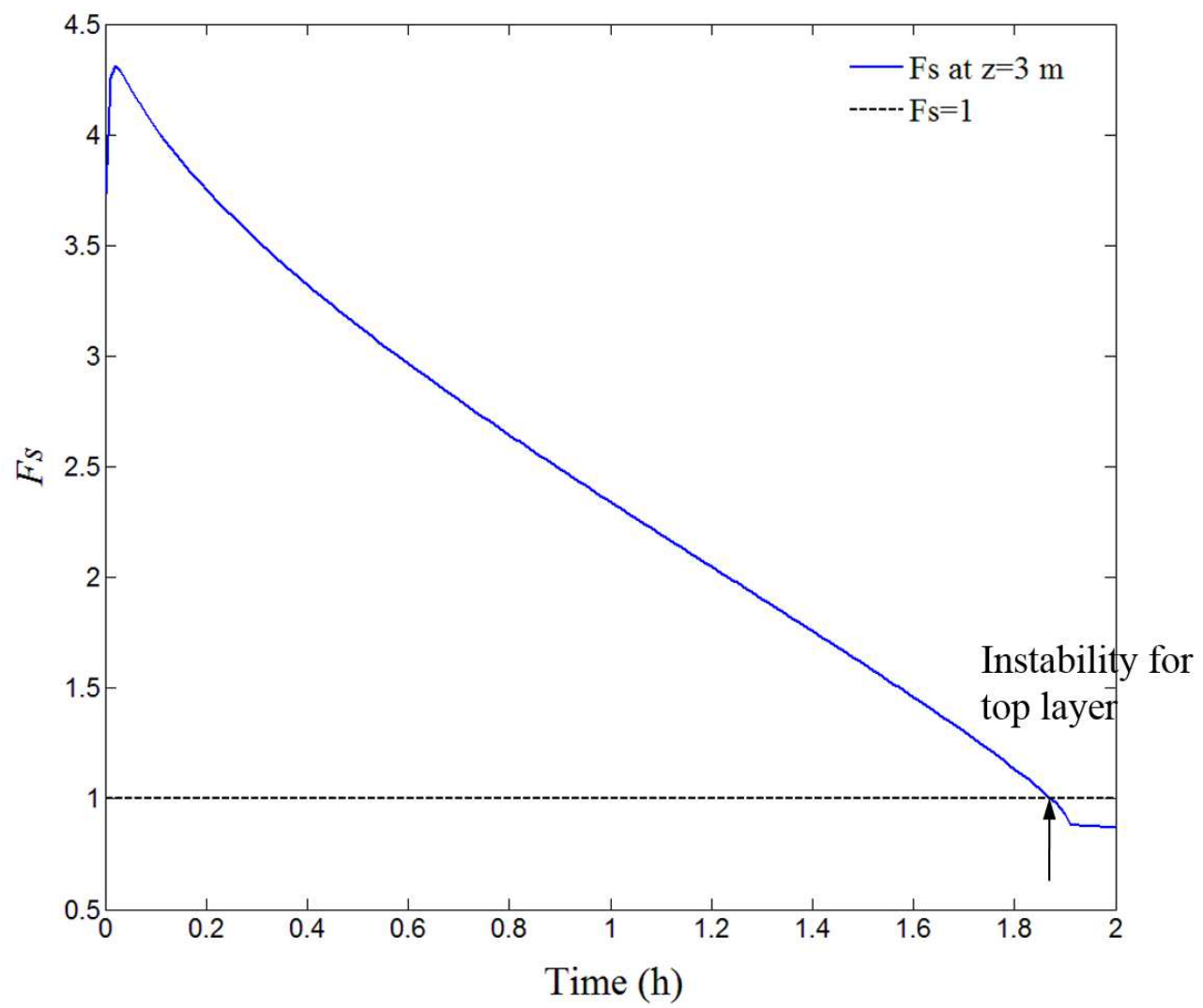

Figure 18. Results of $F_{S}$ at the interface $(z=3 \mathrm{~m})$ for application examples. 


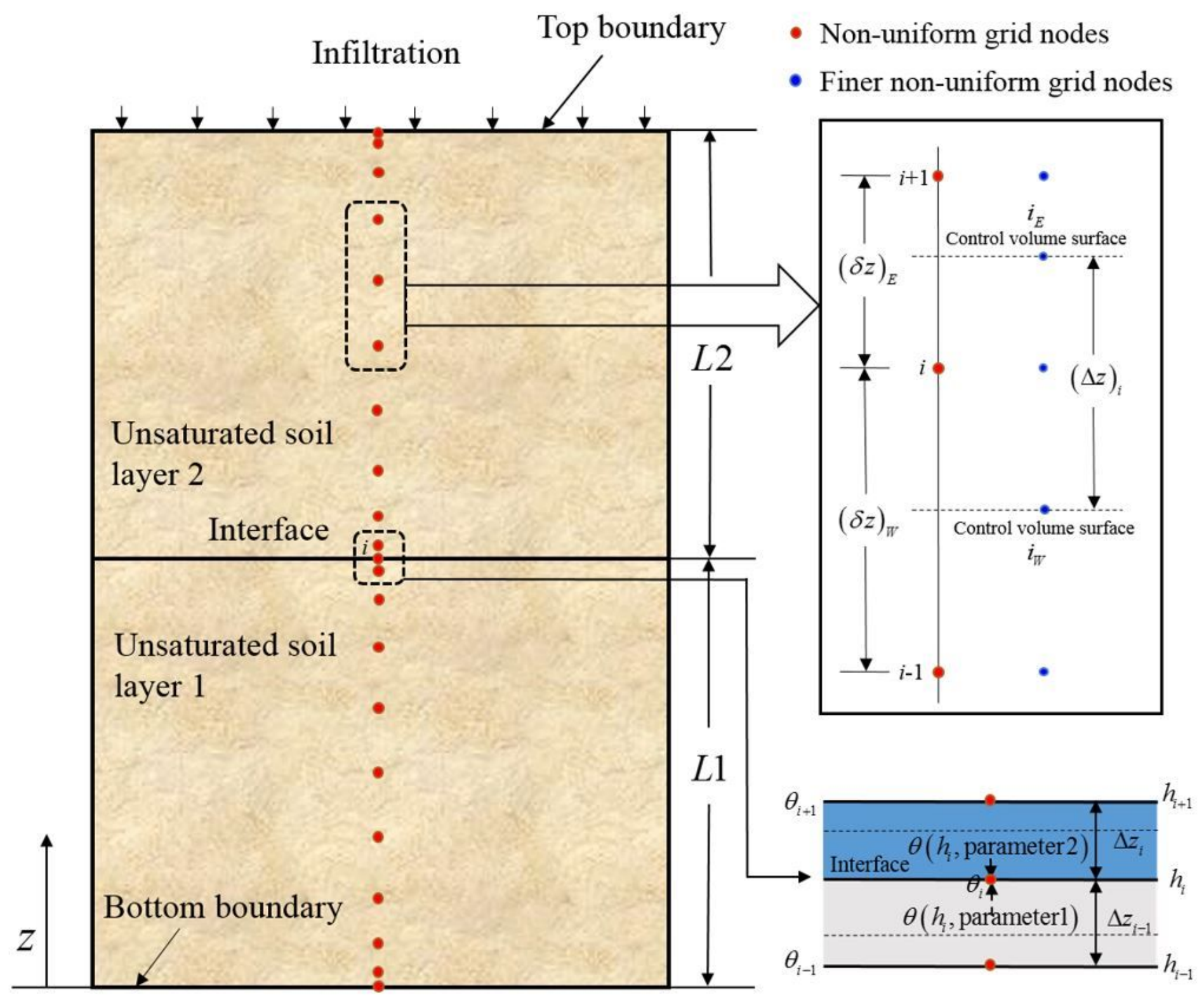

Figure 1

Schematic drawing of control volume method based on non-uniform grid nodes and 1D infiltration model for two-layer soils. 


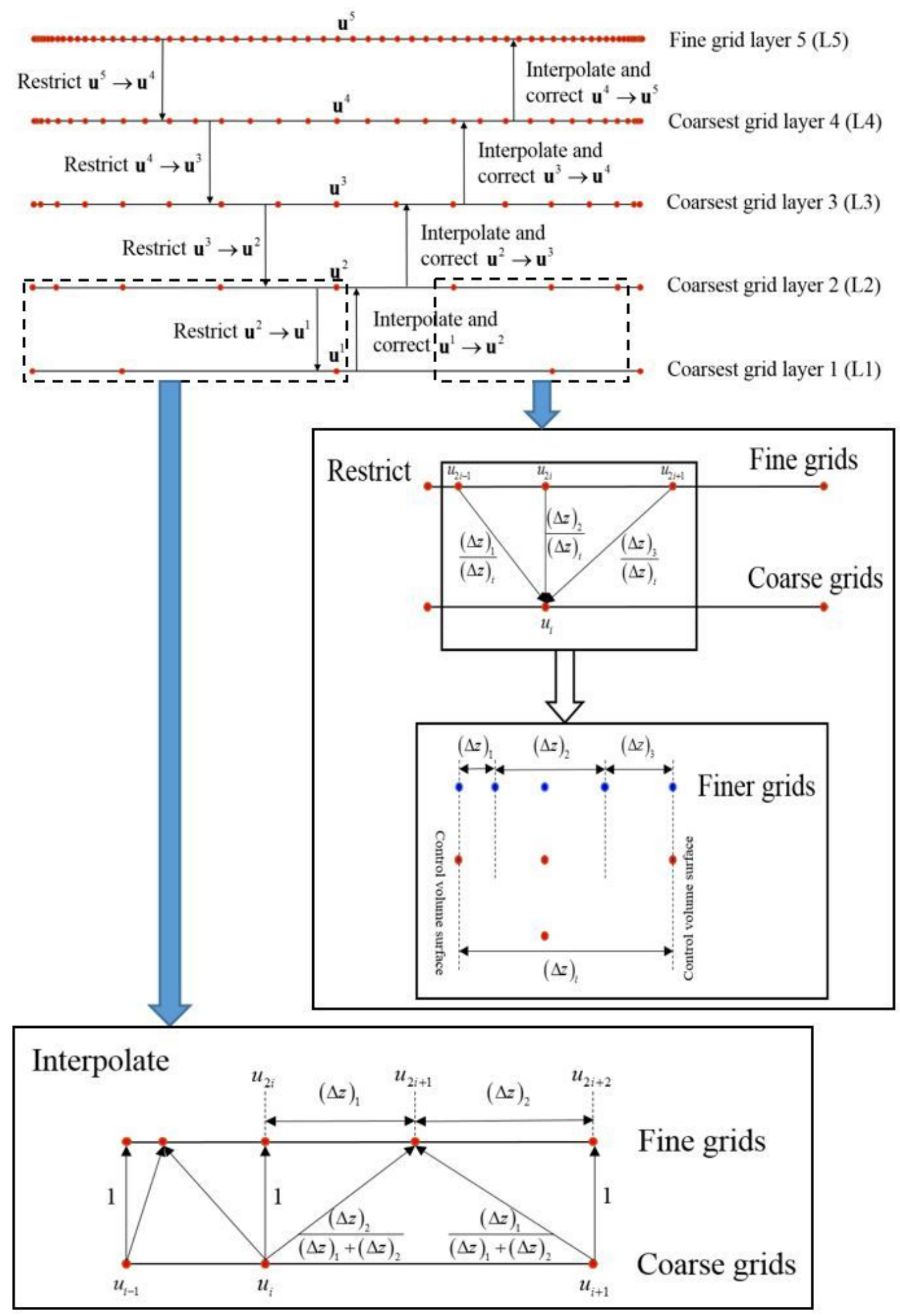

Figure 2

Schematic drawing of non-uniform multigrid correction V-Cycle scheme. 


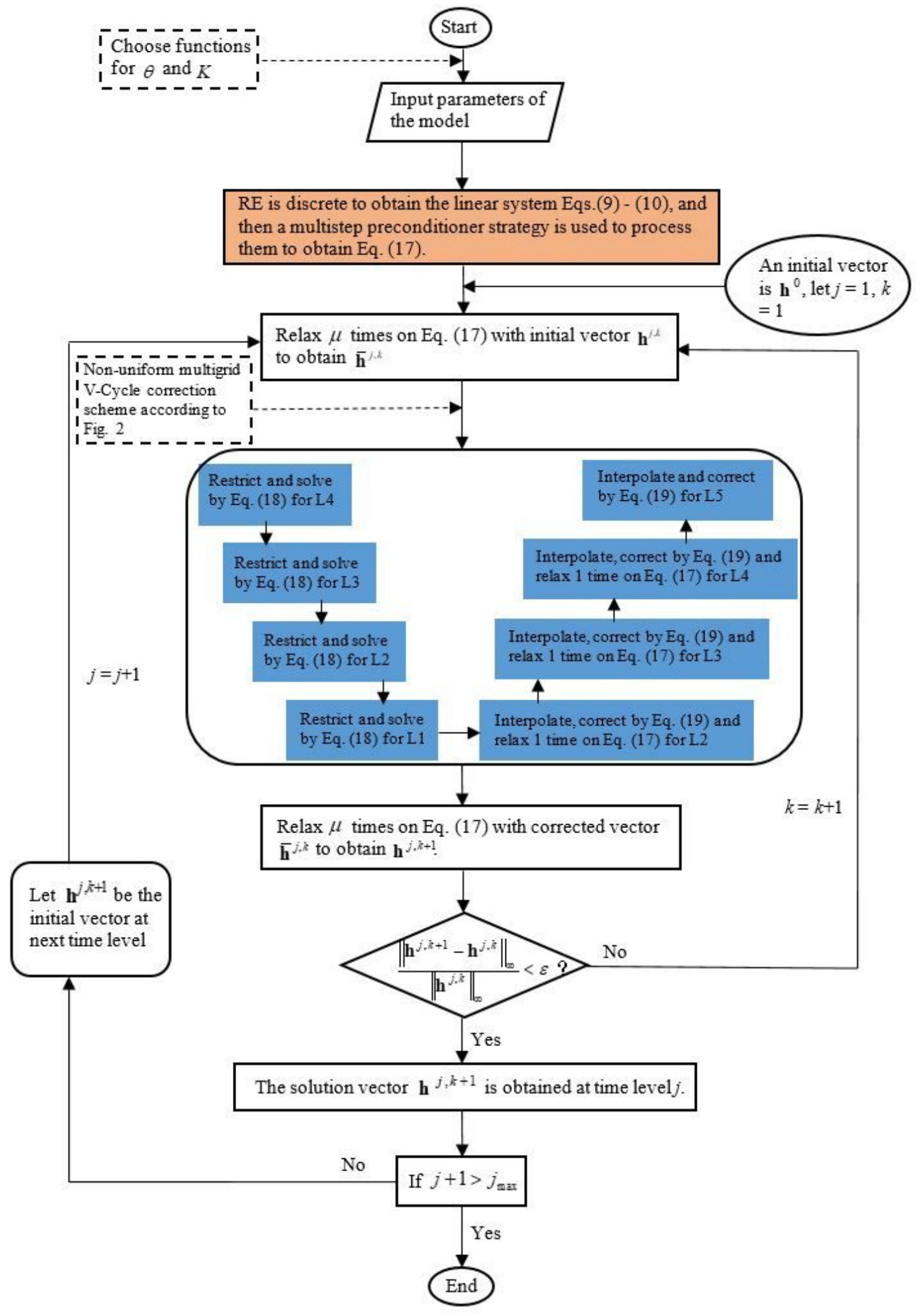

Figure 3

Brief flowchart of the proposed NMG-MPPI(m). 


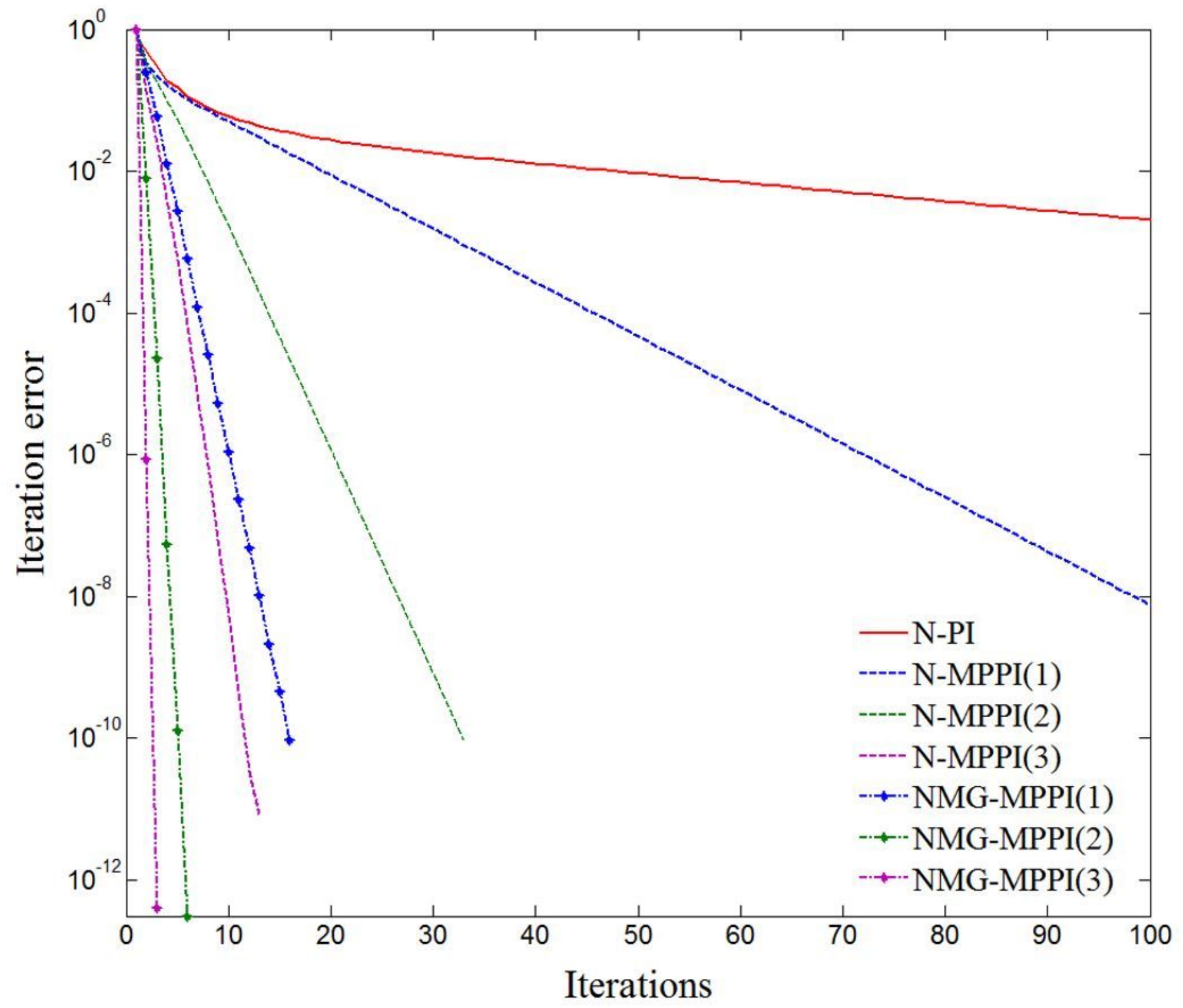

Figure 4

Comparison of convergence rates for the proposed schemes under different number of steps. 


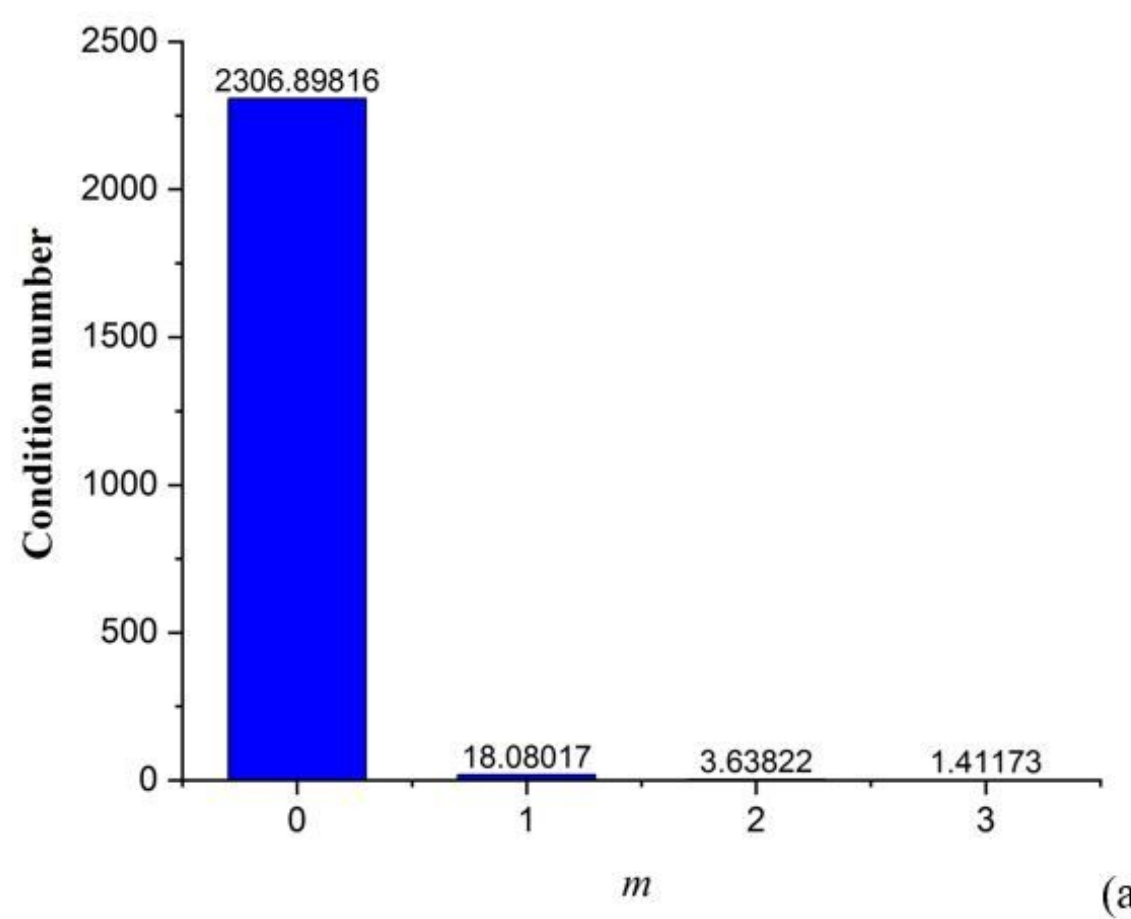

(a)

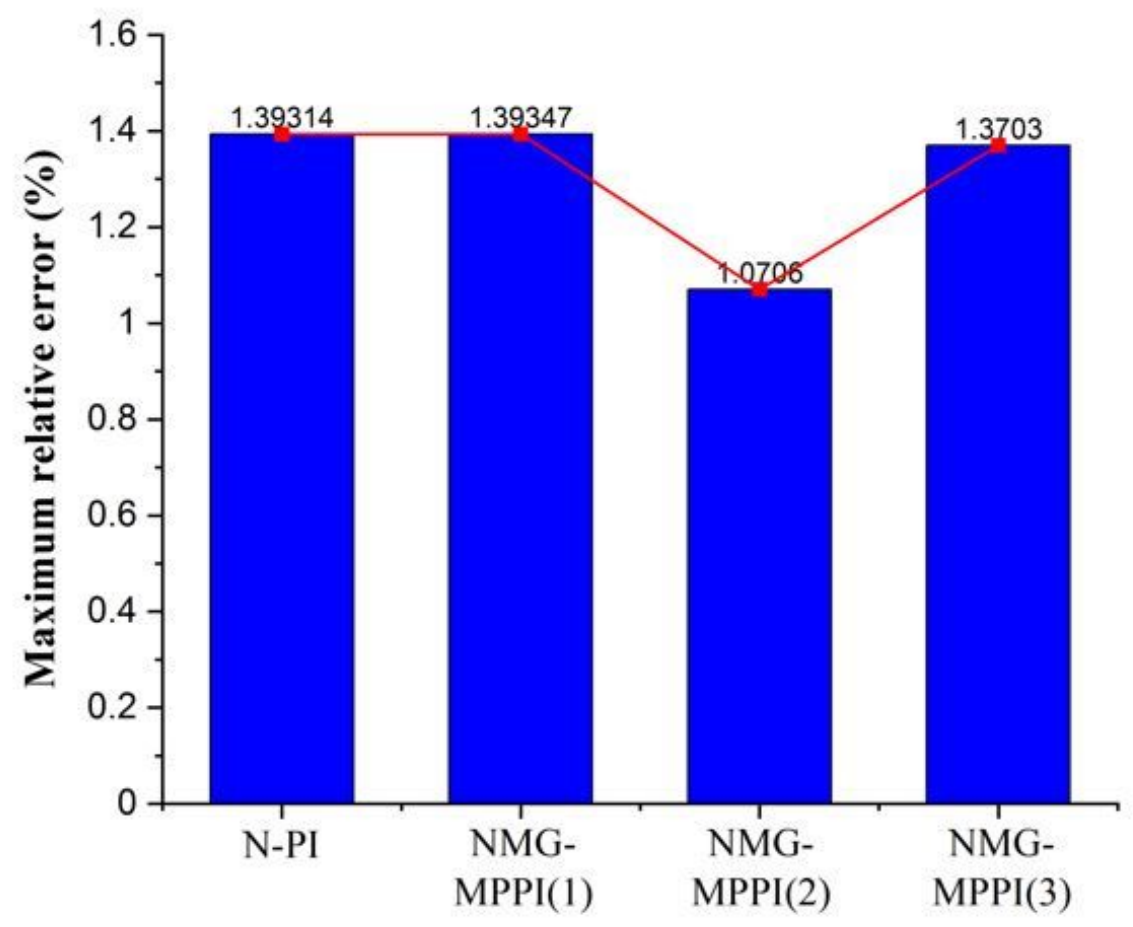

Methods

(b)

Figure 5

Comparison of results for Test 1 over different number of steps using the NMG-MPPI(m) with : (a) condition number; (b) maximum relative error (\%). 


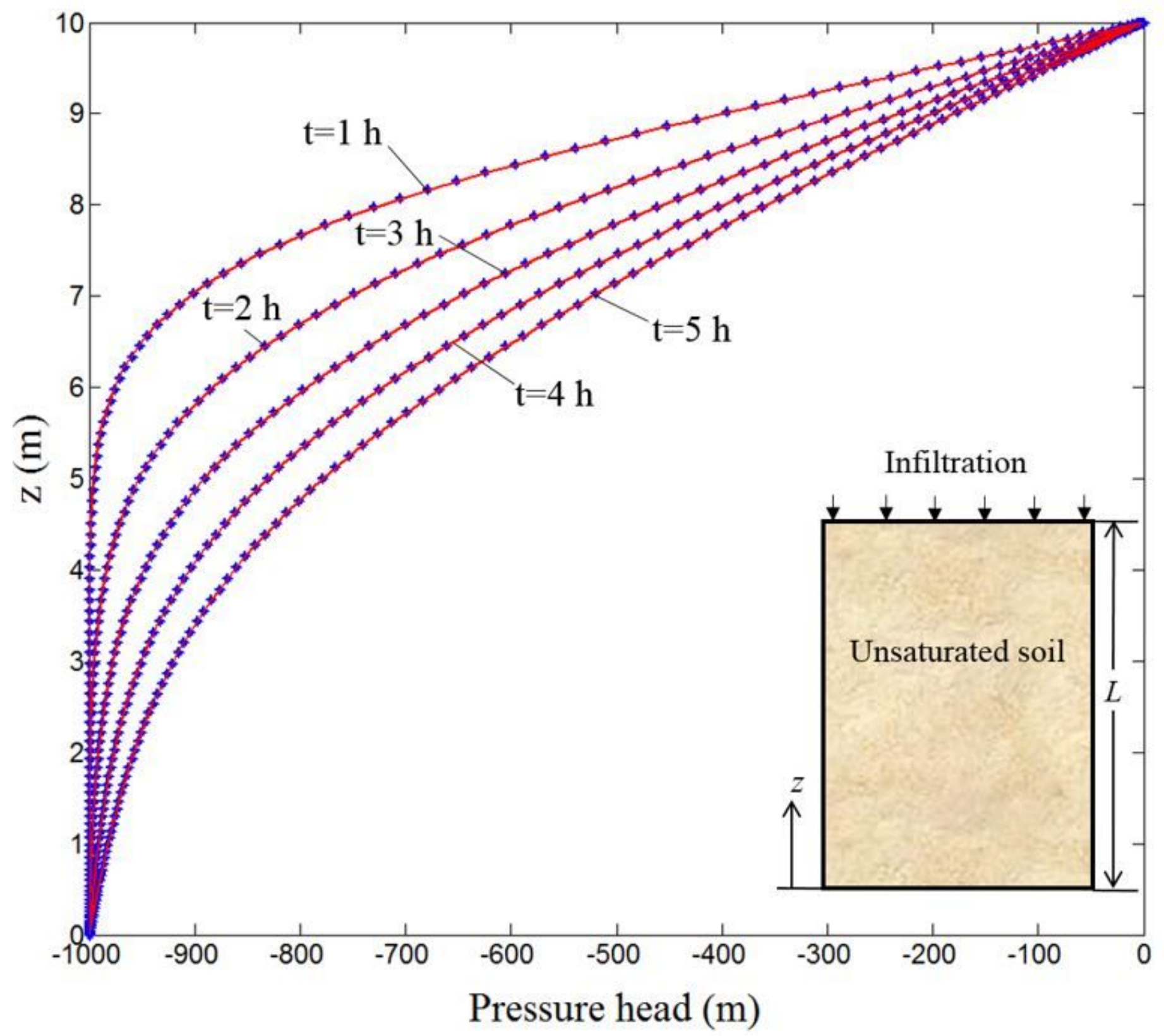

Analytical solution (Tracy, 2006)

*** * Numerical solution by NMG-MPPI(2)

Figure 6

Comparison of the numerical solutions obtained using NMG-MPPI(2) with analytical solutions for Test 1. 


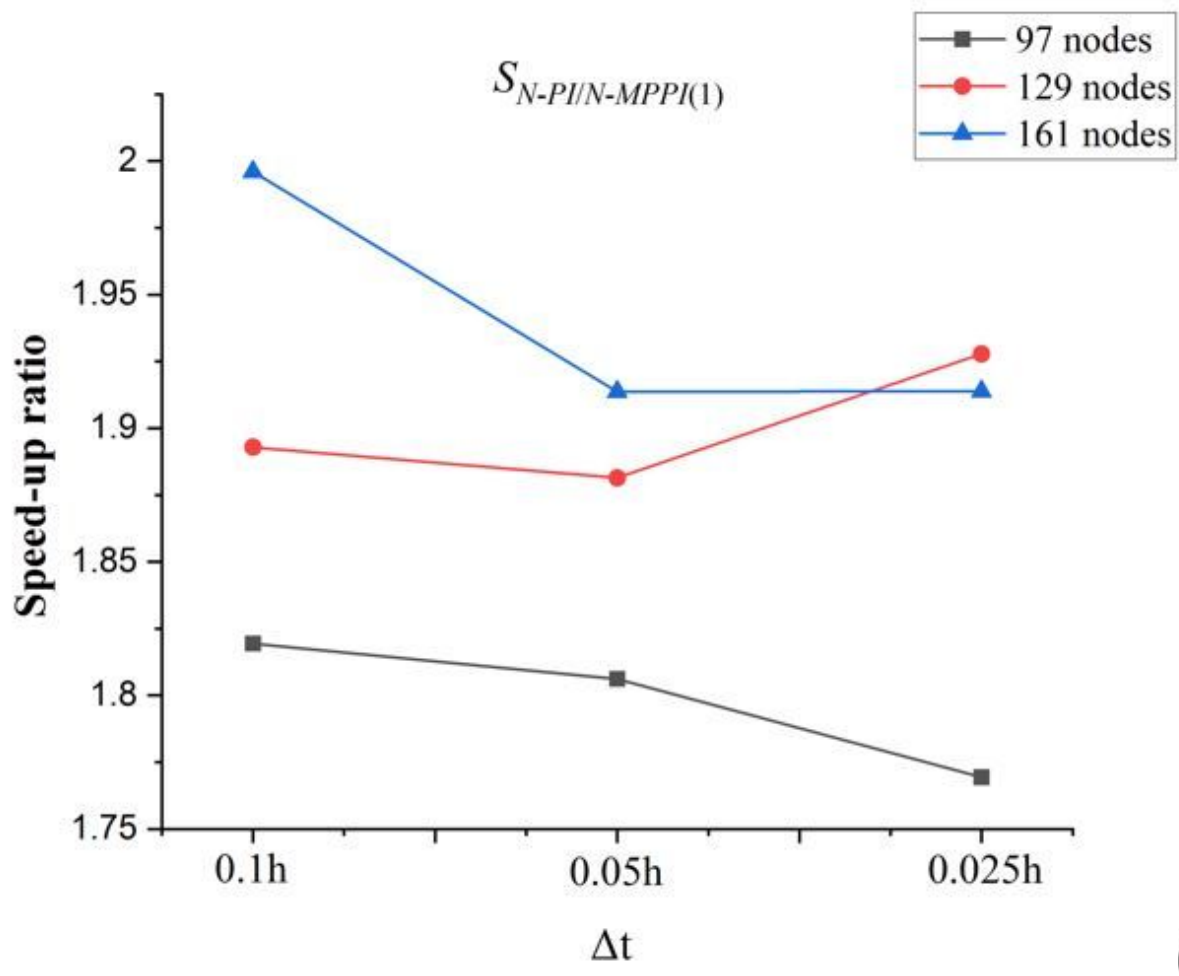

(a)

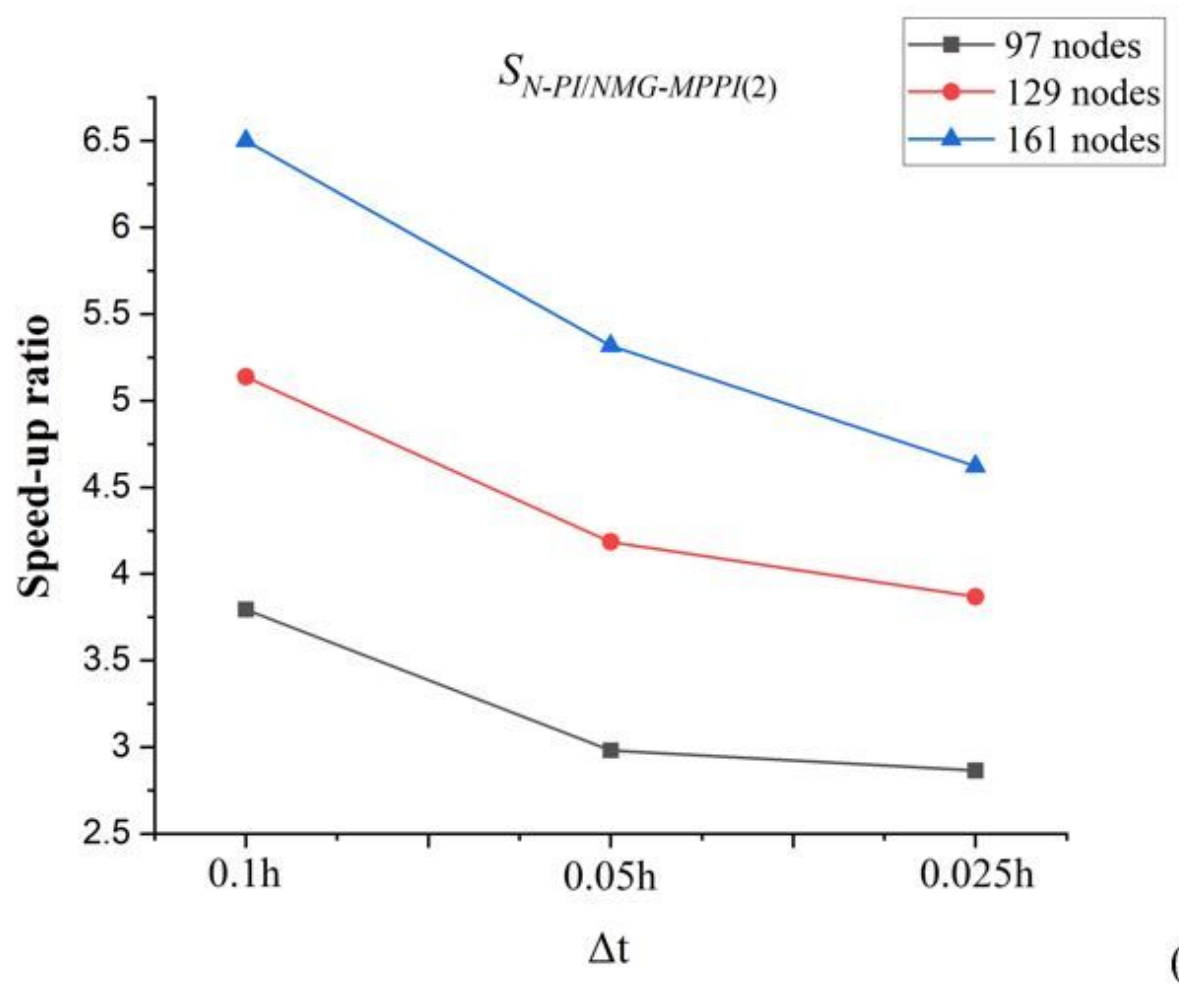

\section{Figure 7}

Comparison of the speed-up ratios at different time steps and number of nodes: (a) SN-PI/N-MPPI(1) ; (b) SN-PI/NMG-MPPI(2) . 


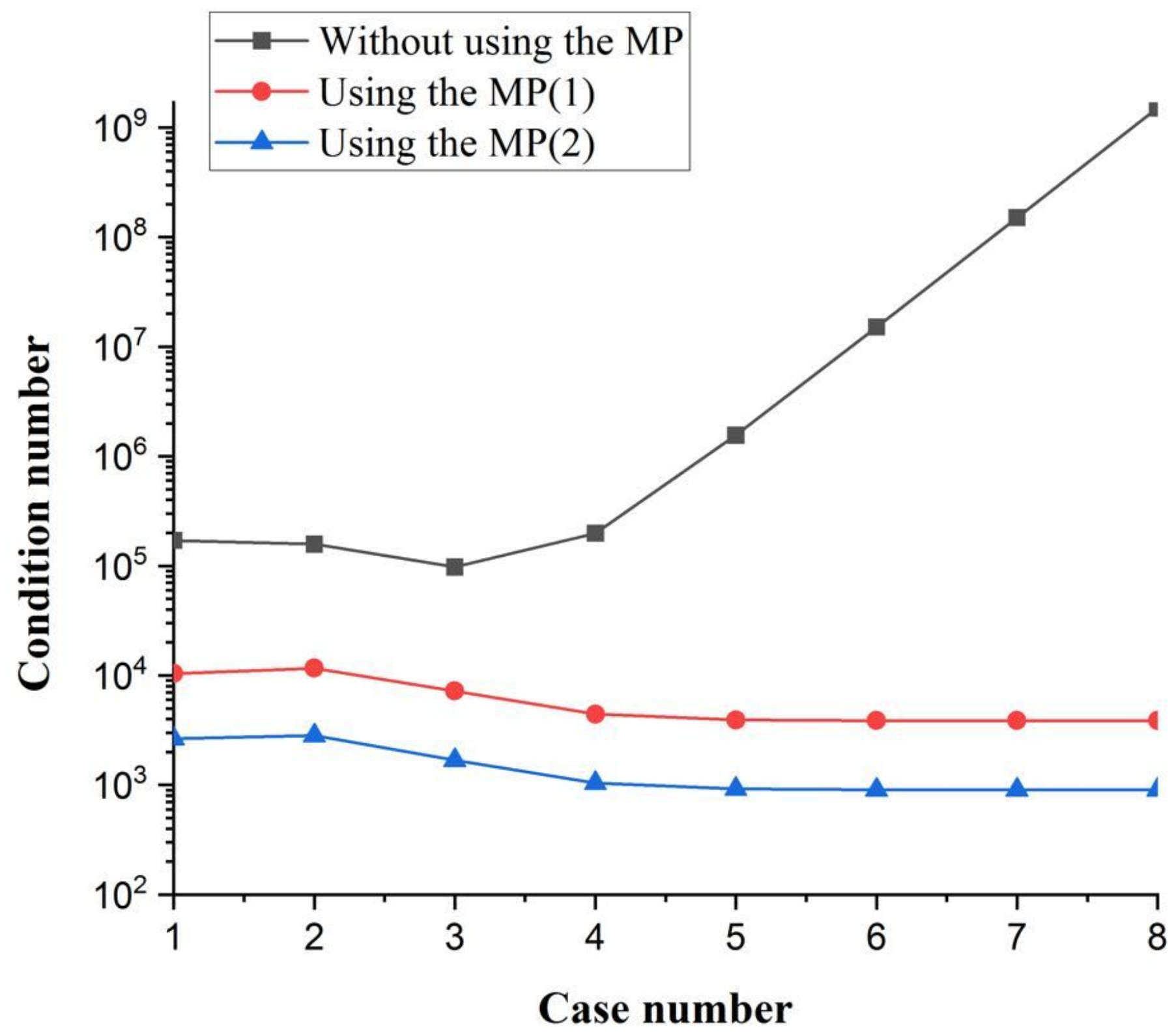

Figure 8

Condition number for Cases 1 to 8 . 


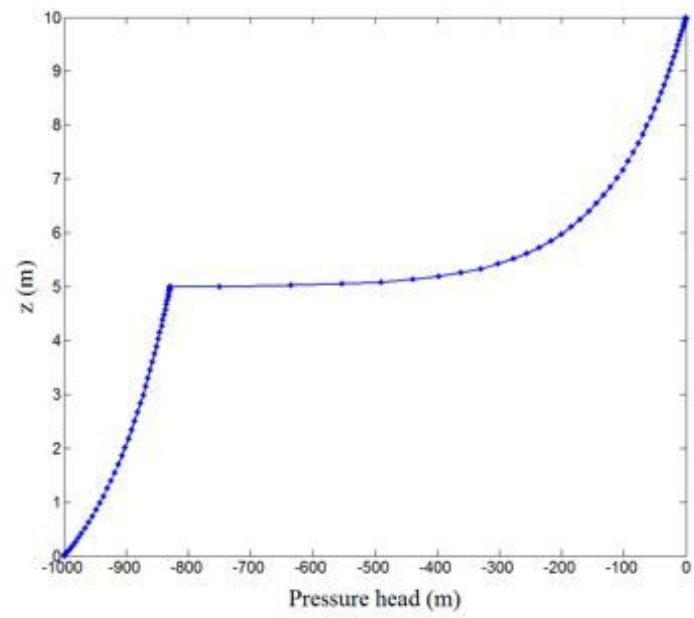

(a)

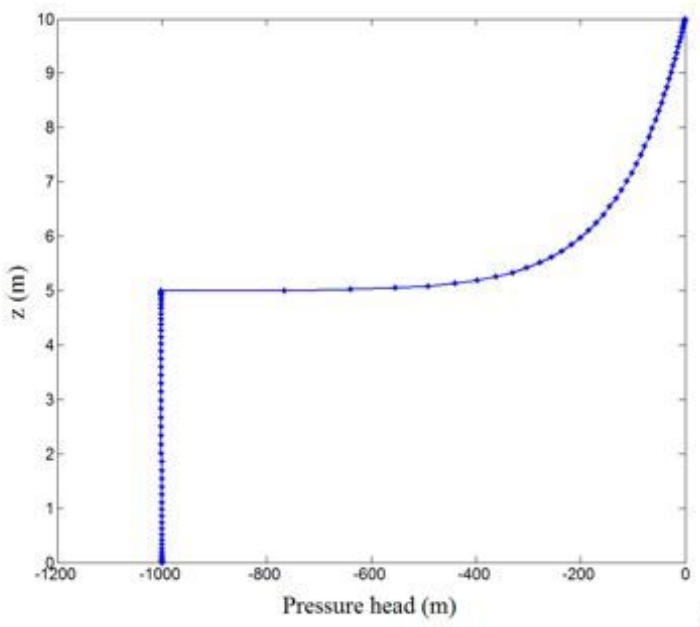

(b)

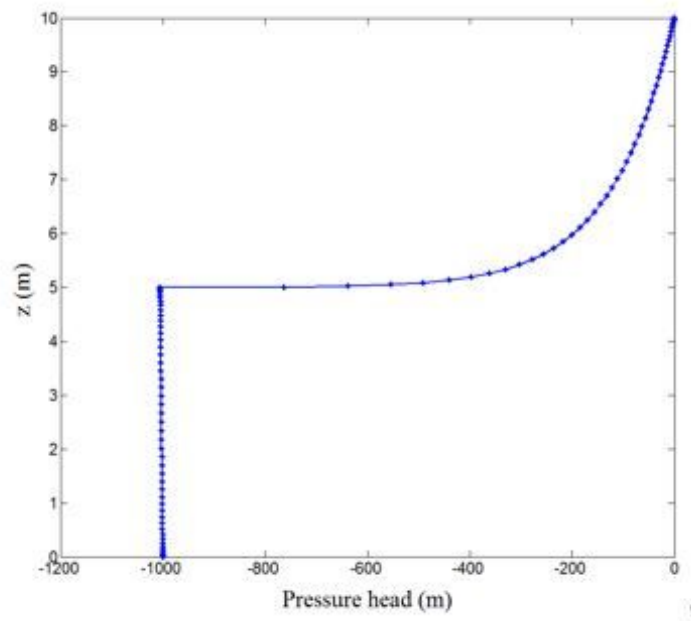

(c)

Figure 9

Numerical solutions obtained using the NMG-MPPI(2): (a) Case 3; (b) Case 5; (c) Case 7. 


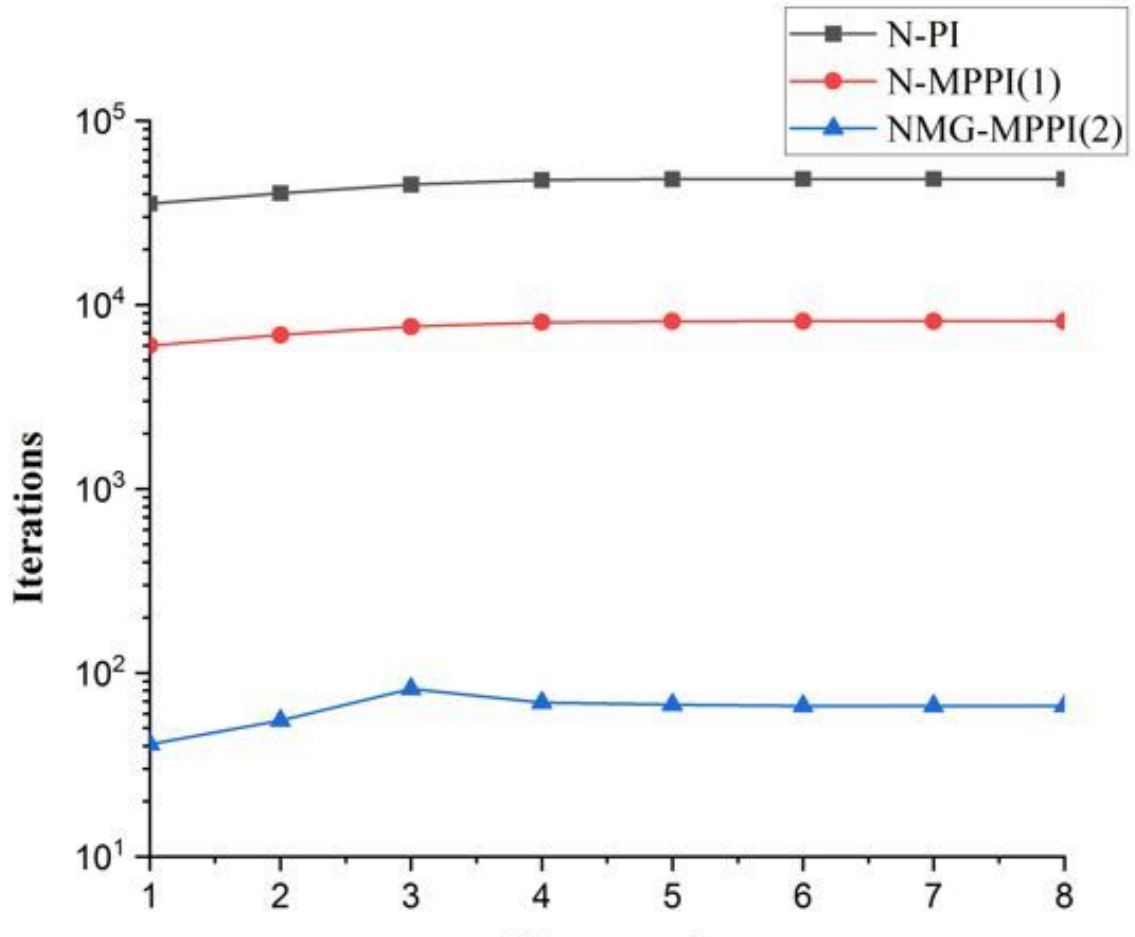

Case number

(a)

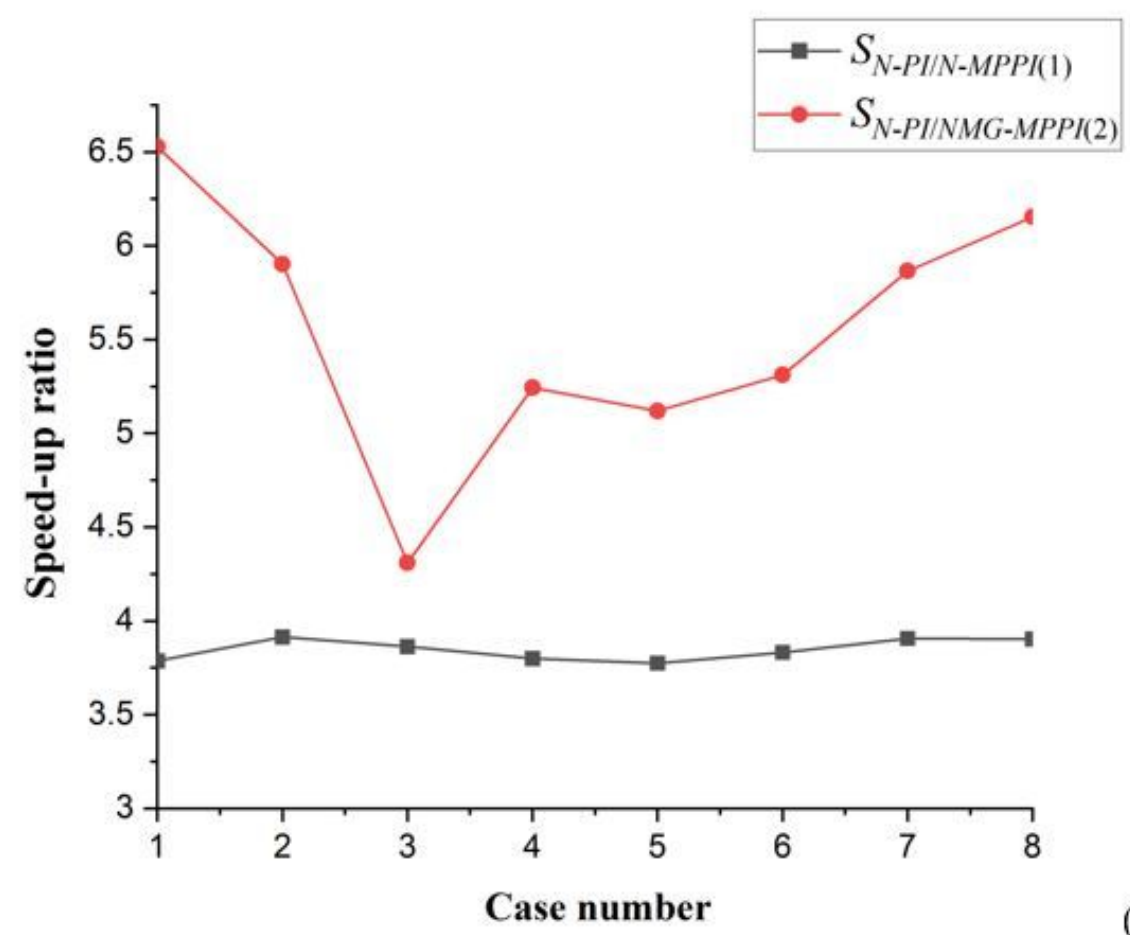

(b)

Figure 10

Numerical results for Cases 1 to 8: (a) Iterations; (b) Speed-up ratio. 


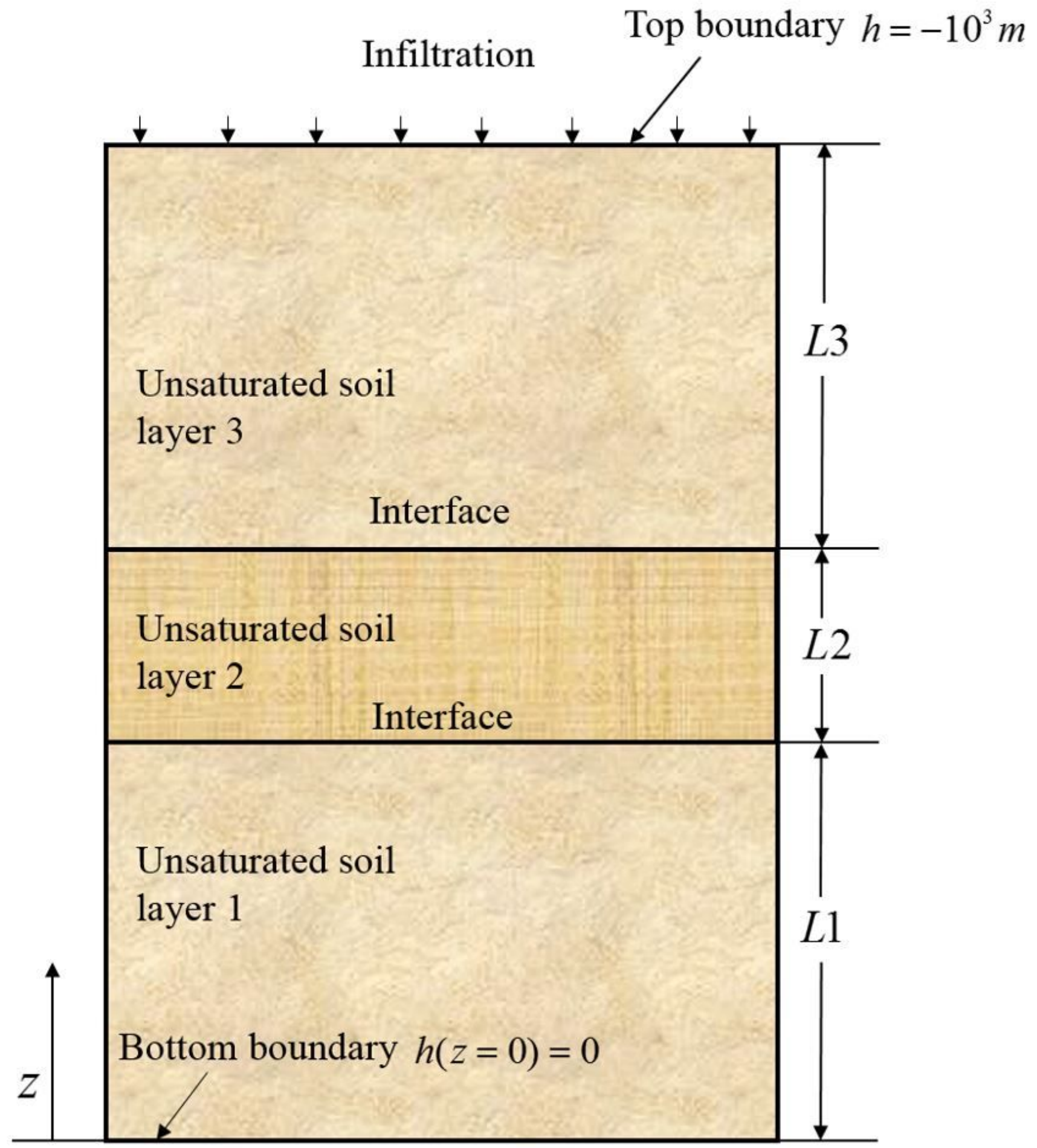

Figure 11

1D infiltration model for three-layer soils. 


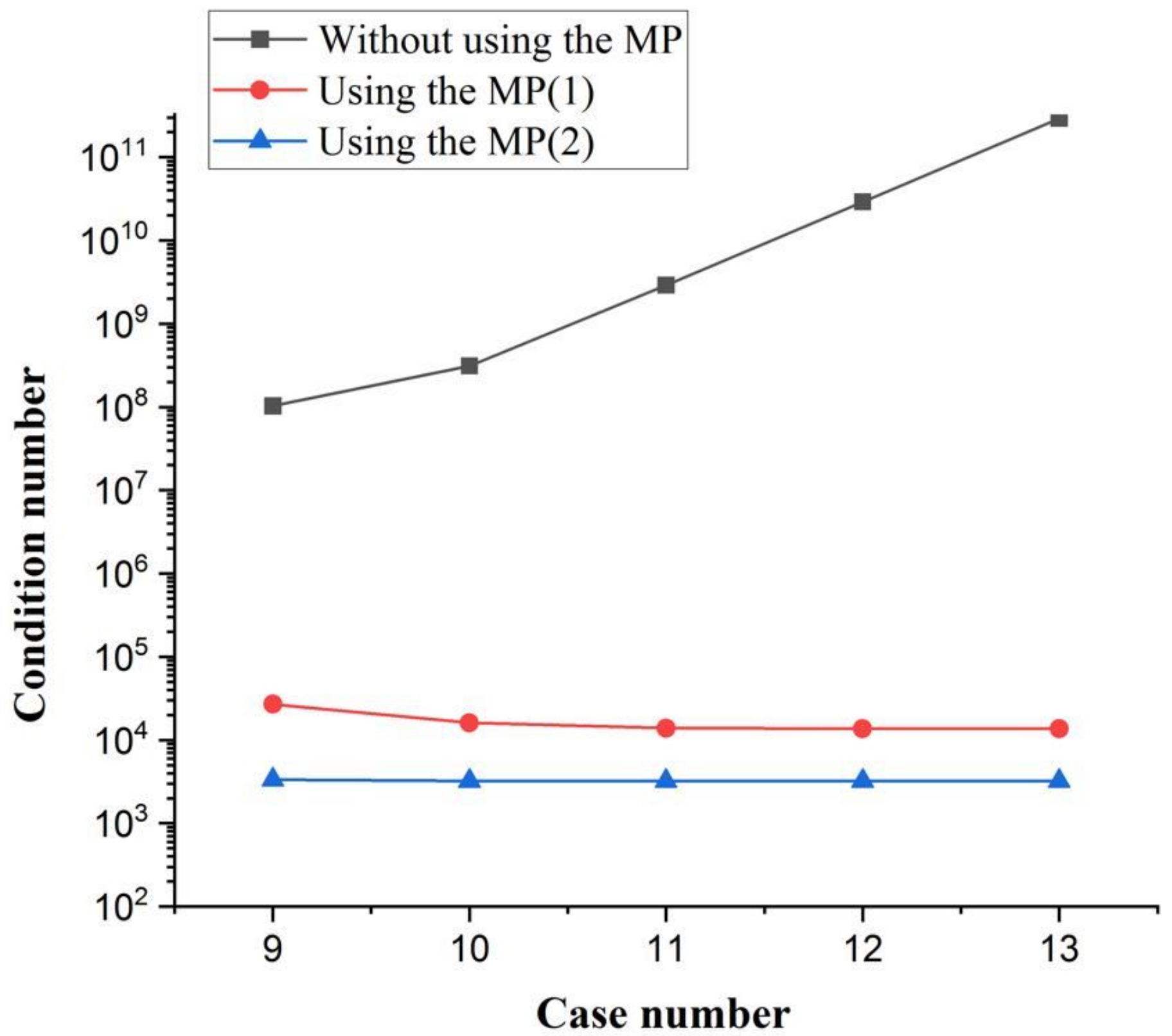

Figure 12

Condition number for Cases 9 to 13 . 


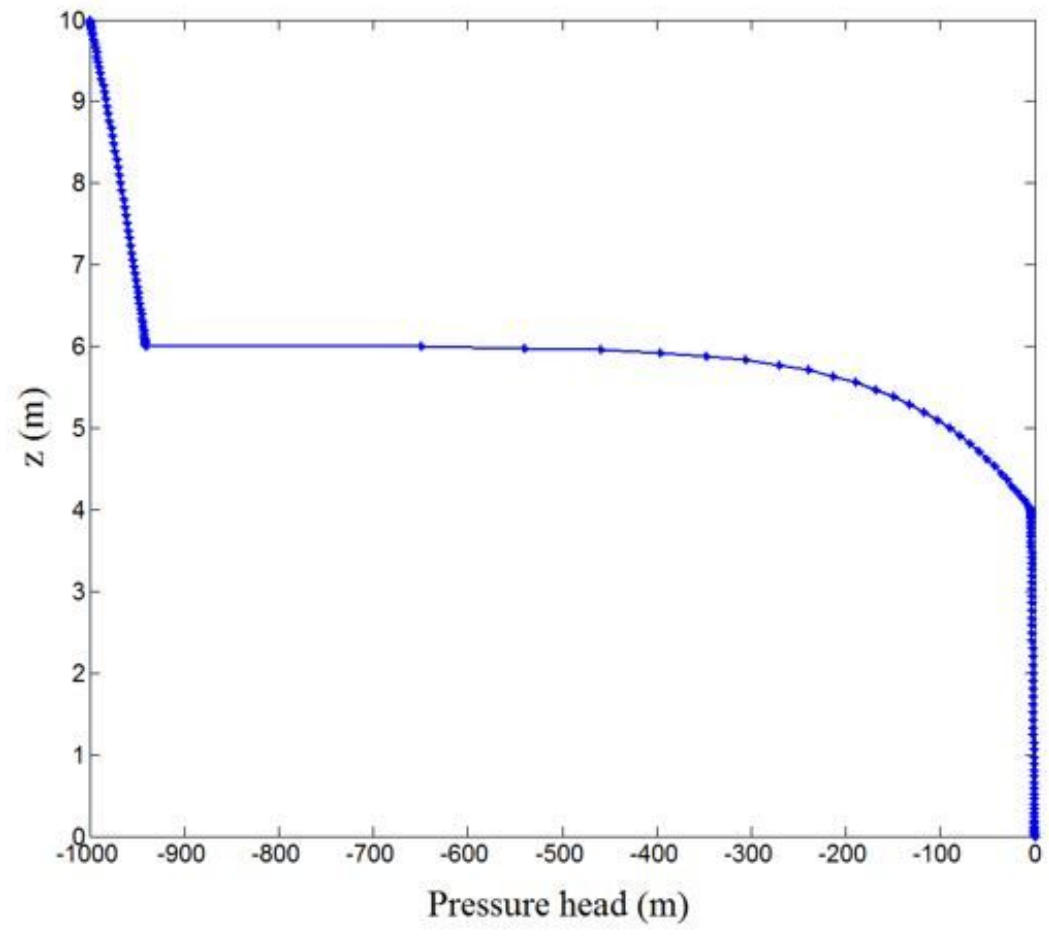

(a)

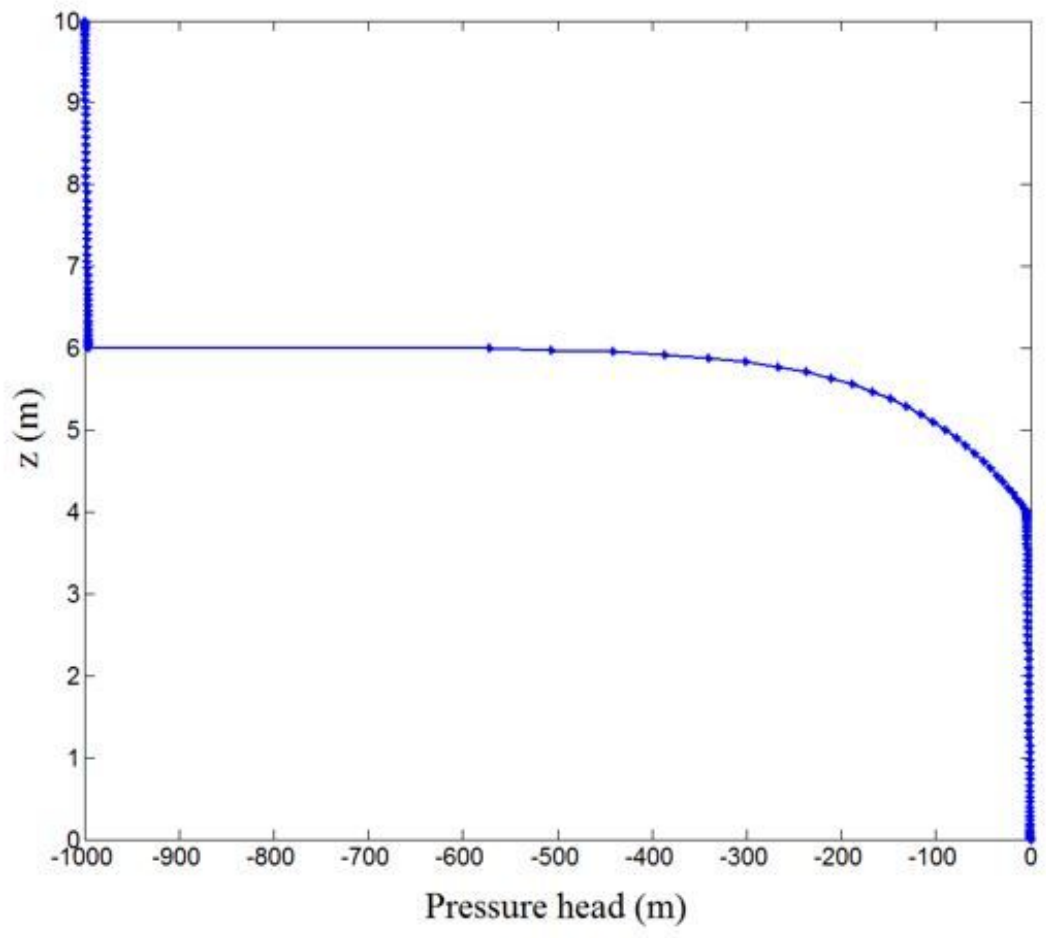

(b)

Figure 13

Numerical solutions obtained using the NMG-MPPI(2): (a) Case 10; (b) Case 12. 


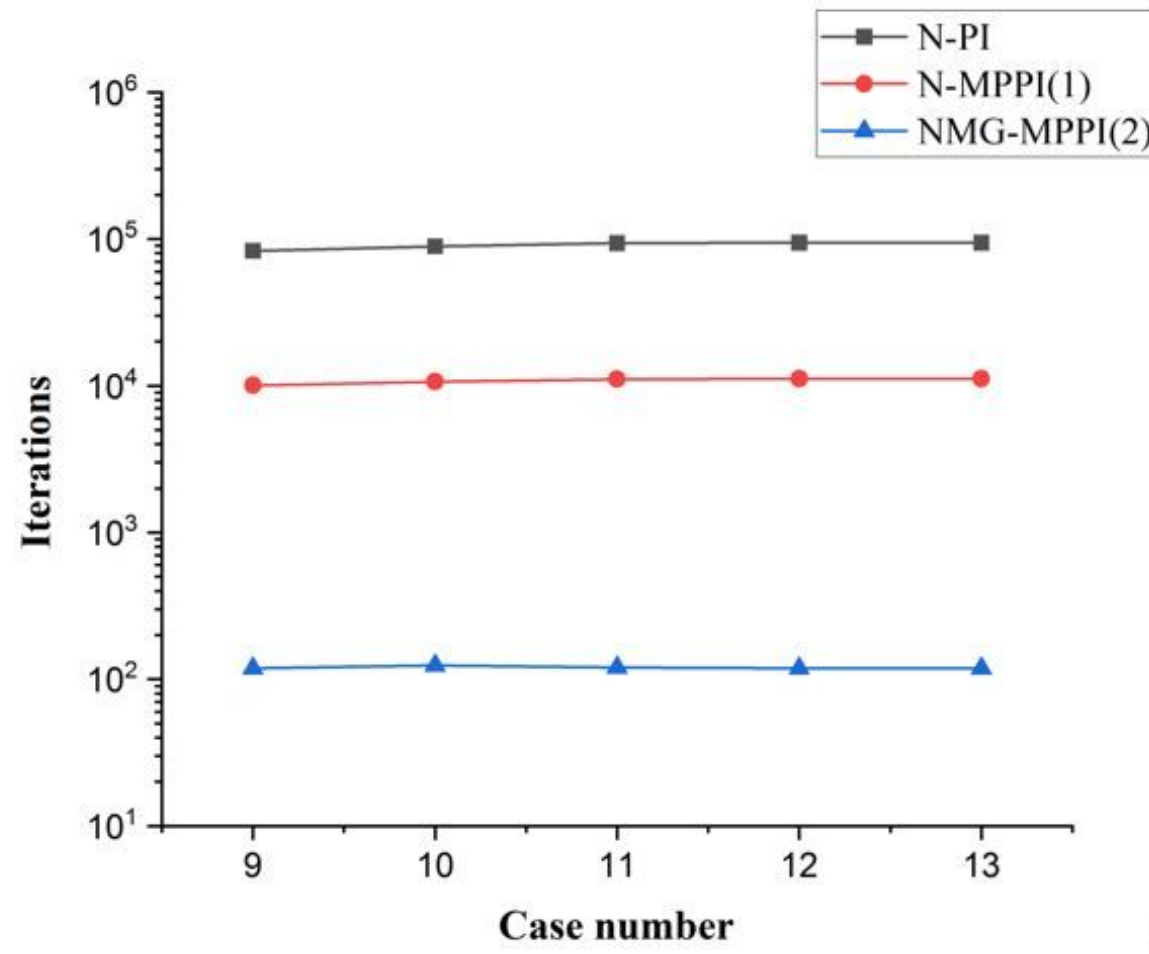

(a)

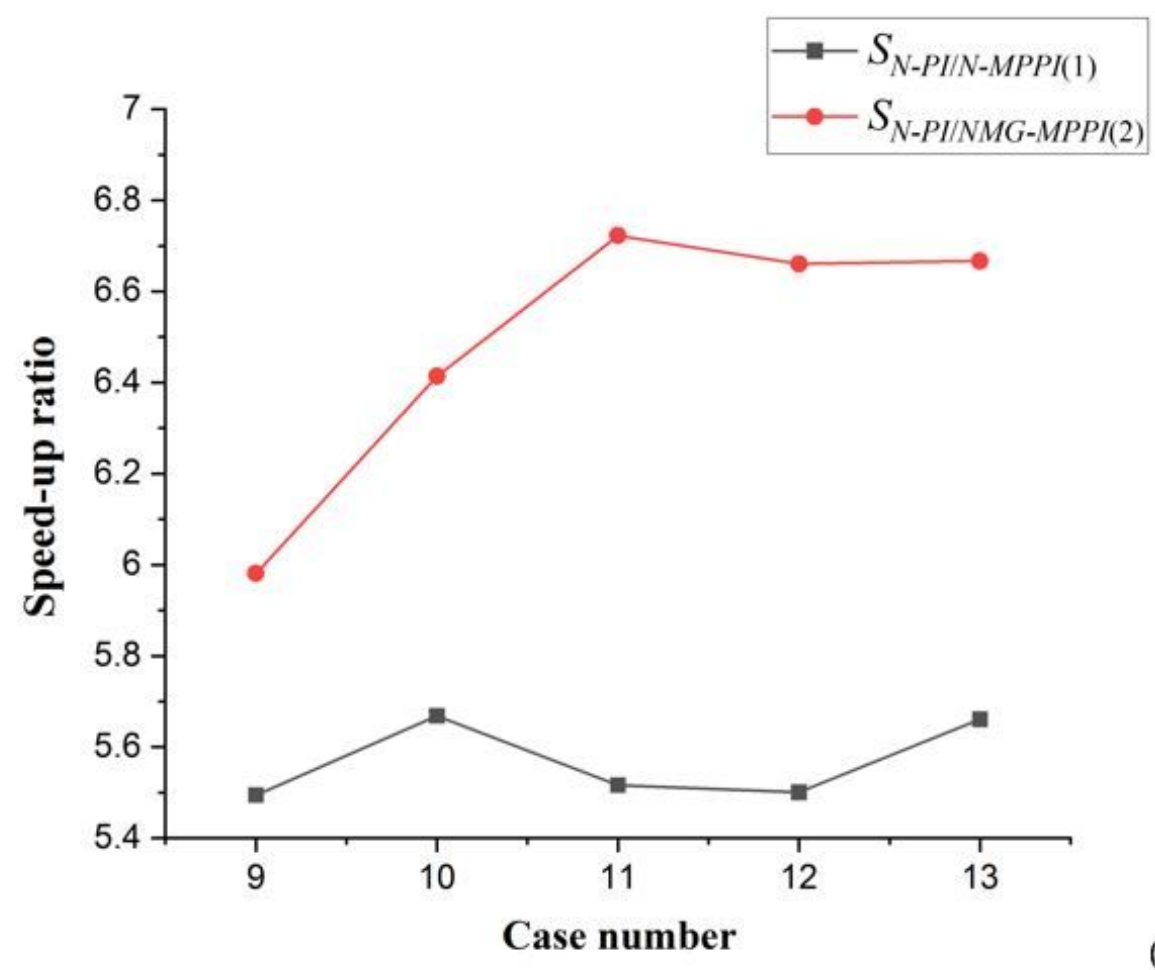

Figure 14

Numerical results for Cases 9 to 13: (a) Iterations; (b) Speed-up ratio. 


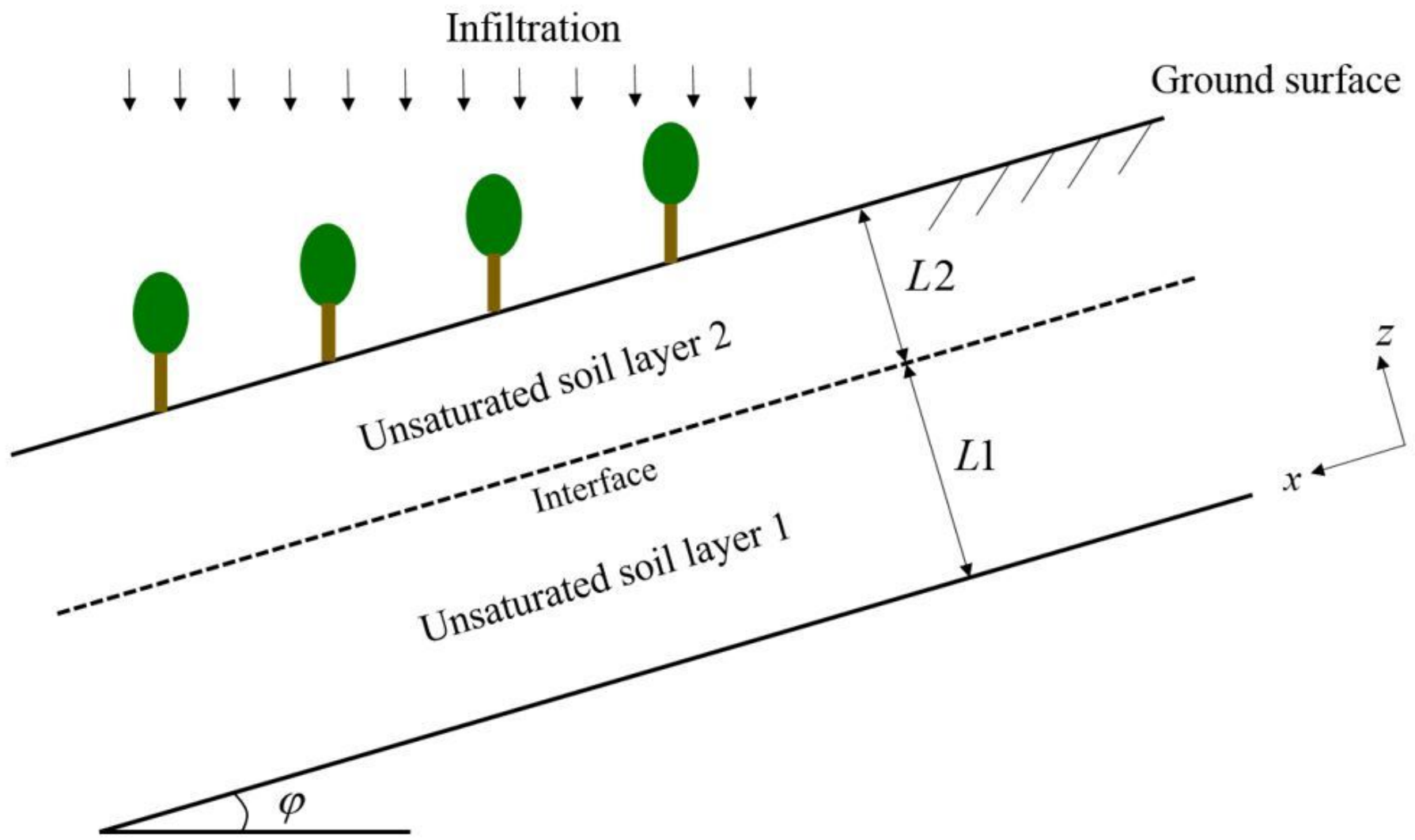

Figure 15

Schematic diagram for two-layer soil slopes. 


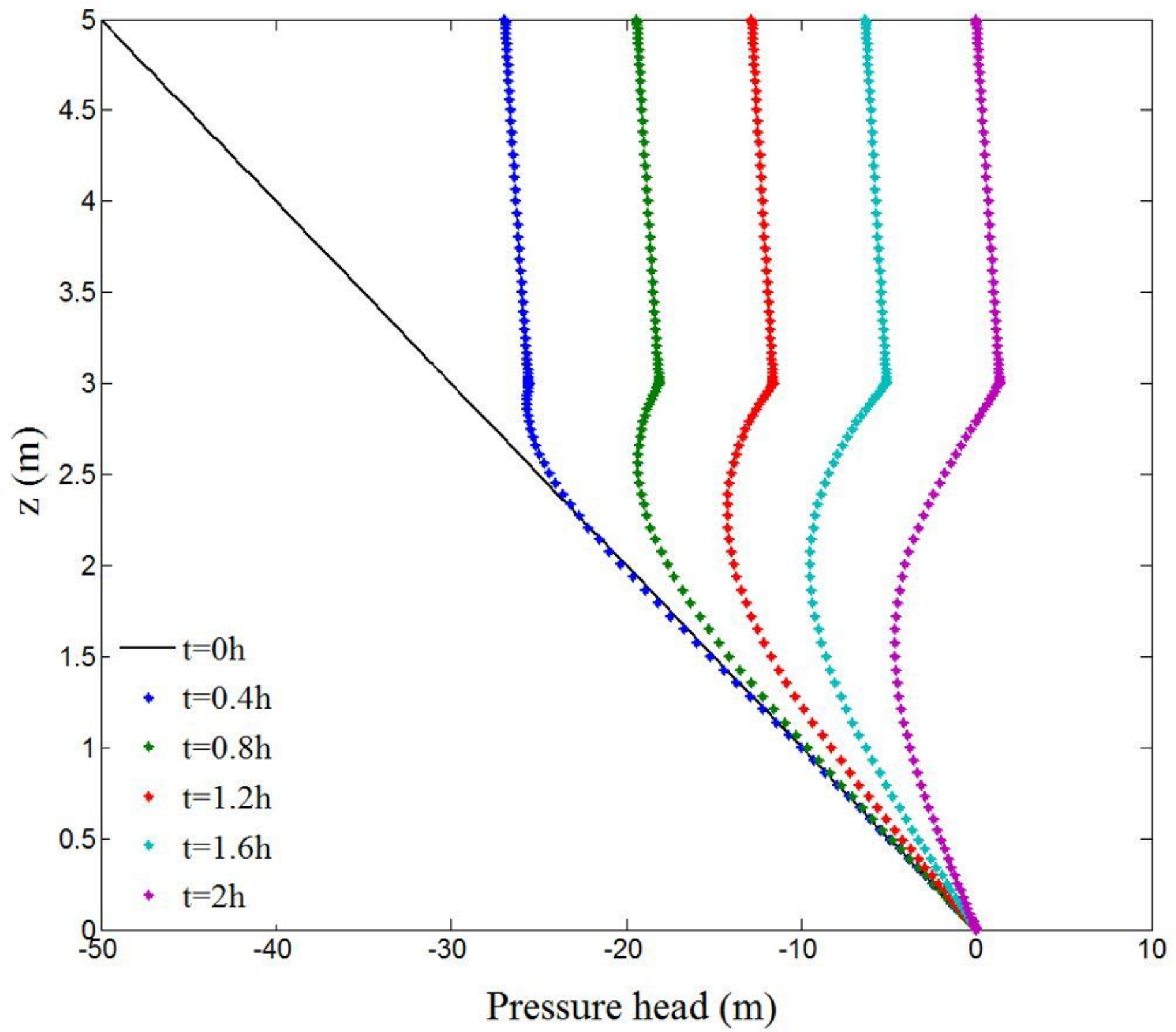

Figure 16

Computed profile of pressure head for application examples. 


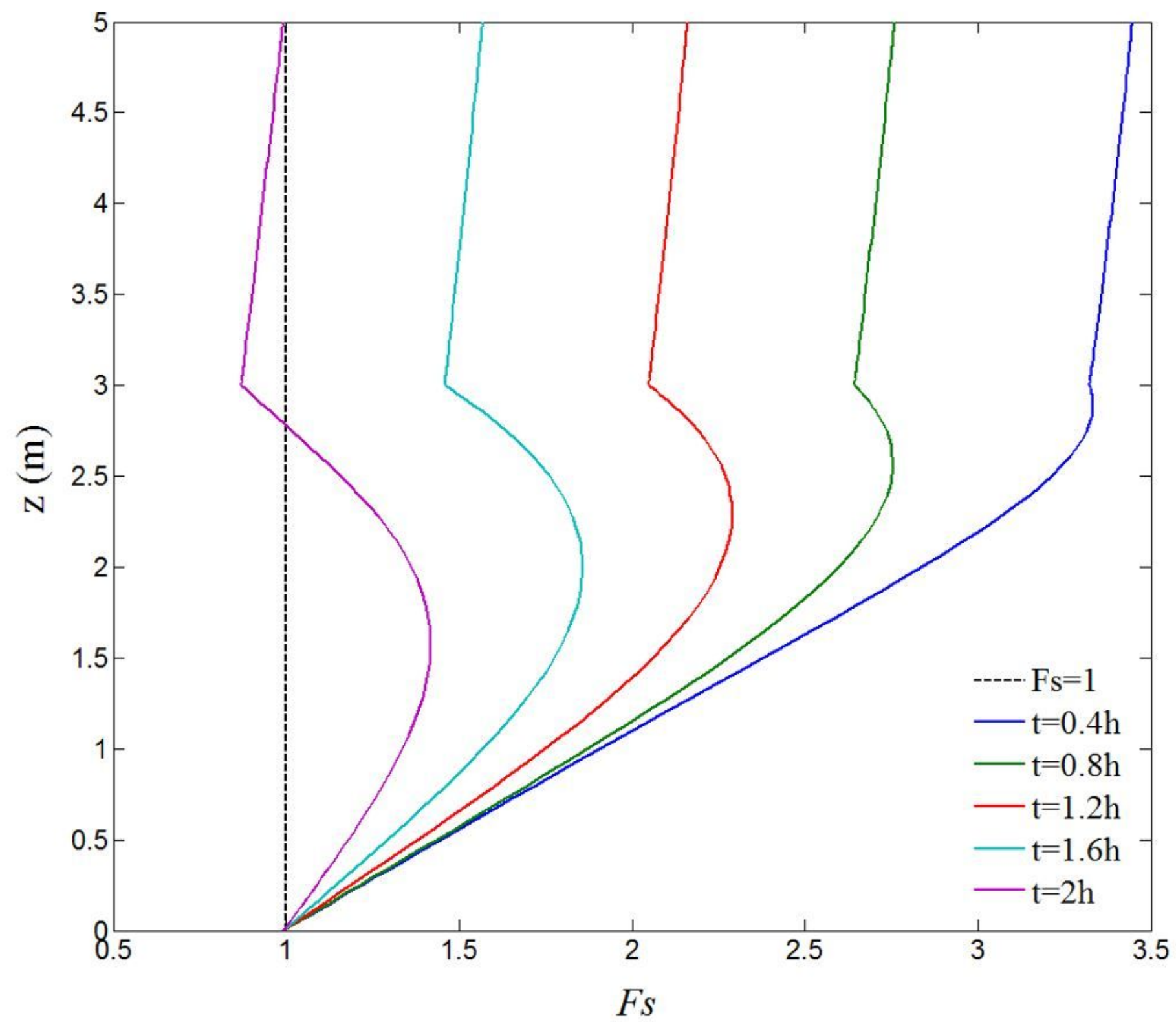

Figure 17

Computed profile of FS for application examples. 


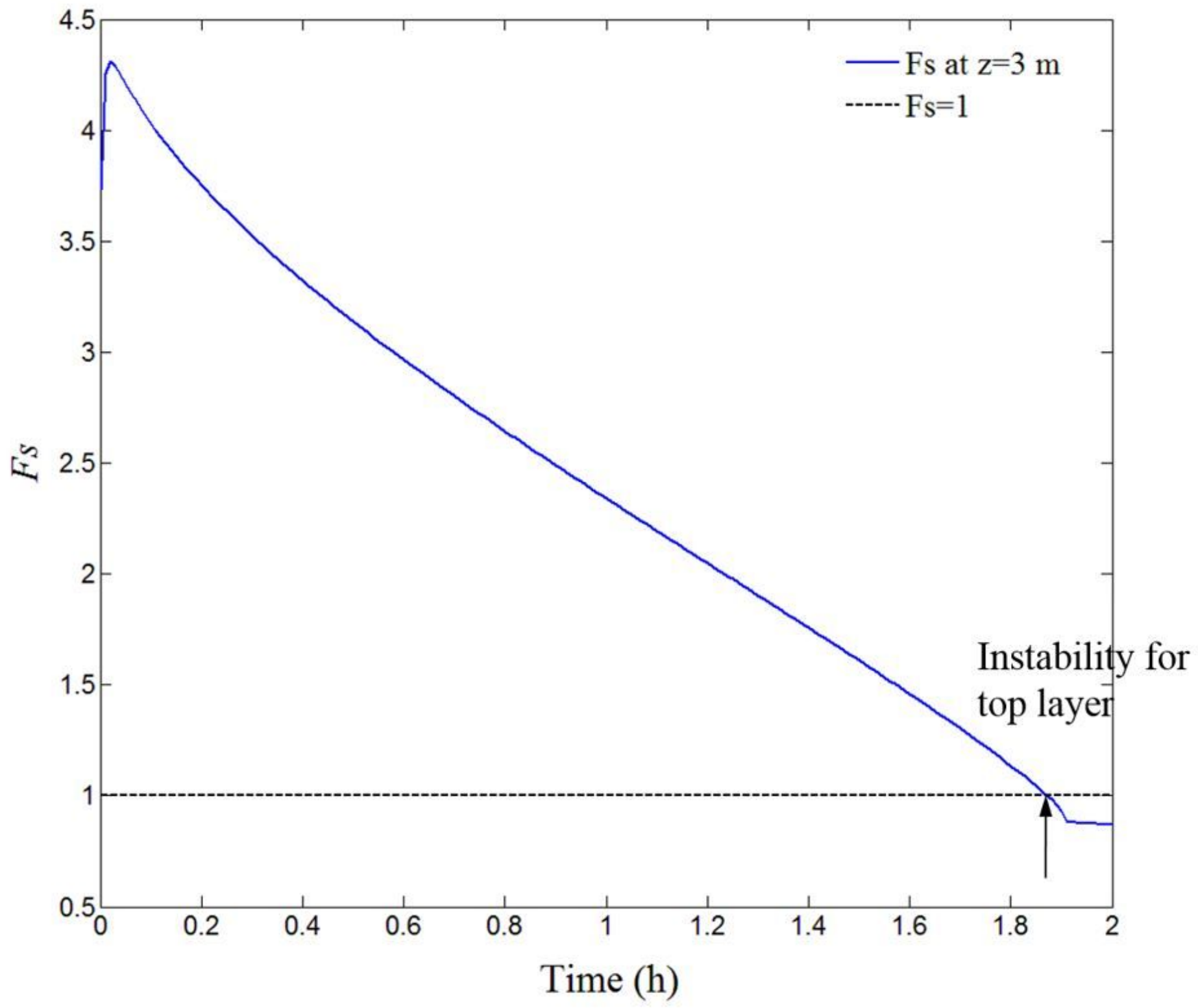

Figure 18

Results of FS at the interface $(z=3 m)$ for application examples. 